ARTICLE

\title{
A critical role of PRDM14 in human primordial germ cell fate revealed by inducible degrons
}

\author{
Anastasiya Sybirna, ${ }^{1,2}$, , Walfred W.C. Tang 1,2, Merrick Pierson Smela (1) 1,2, Sabine Dietmann², \\ Wolfram H. Gruhn ${ }^{1,2}$, Ran Brosh (D) ${ }^{4} \&$ M. Azim Surani (1, 1,3凶
}

PRDM14 is a crucial regulator of mouse primordial germ cells (mPGCs), epigenetic reprogramming and pluripotency, but its role in the evolutionarily divergent regulatory network of human PGCs (hPGCs) remains unclear. Besides, a previous knockdown study indicated that PRDM14 might be dispensable for human germ cell fate. Here, we decided to use inducible degrons for a more rapid and comprehensive PRDM14 depletion. We show that PRDM14 loss results in significantly reduced specification efficiency and an aberrant transcriptome of hPGC-like cells (hPGCLCs) obtained in vitro from human embryonic stem cells (hESCs). Chromatin immunoprecipitation and transcriptomic analyses suggest that PRDM14 cooperates with TFAP2C and BLIMP1 to upregulate germ cell and pluripotency genes, while repressing WNT signalling and somatic markers. Notably, PRDM14 targets are not conserved between mouse and human, emphasising the divergent molecular mechanisms of PGC specification. The effectiveness of degrons for acute protein depletion is widely applicable in various developmental contexts.

\footnotetext{
${ }^{1}$ Wellcome Trust/Cancer Research UK Gurdon Institute, Henry Wellcome Building of Cancer and Developmental Biology, Cambridge CB2 1QN, UK.

${ }^{2}$ Physiology, Development and Neuroscience Department, University of Cambridge, Cambridge CB2 3EL, UK. ${ }^{3}$ Wellcome Trust/Medical Research Council Cambridge Stem Cell Institute, University of Cambridge, Cambridge CB2 1QR, UK. ${ }^{4}$ Institute for Systems Genetics, NYU Langone Health, New York, NY 10016, USA. ${ }_{\text {email: a.surani@gurdon.cam.ac.uk }}$
} 
G ametes develop from primordial germ cells (PGCs), the embryonic precursors, which are apparently specified in approximately week $2-3$ human embryos ${ }^{1,2}$. While the requirement for BMP and WNT signalling for PGC specification is conserved between mouse and human ${ }^{3-5}$, the gene regulatory network for hPGC fate has diverged ${ }^{6}$. Mouse PGCs (mPGCs) are specified by three core transcription factors (TFs): $\operatorname{Prdm} 1$ (encoding BLIMP1), Prdm14, and Tfap2c (encoding AP2 $\gamma$ ) 7,8 , among which PRDM14 plays a central role; loss of Prdm14 abrogates mPGC specification ${ }^{9}$, while its overexpression is sufficient to induce $\mathrm{mPGC}$ fate in vitro ${ }^{8}$.

During $\mathrm{mPGC}$ specification, PRDM14 induces upregulation of germline-specific genes, assists BLIMP1-mediated repression of somatic transcripts and initiates global epigenetic reprogramming ${ }^{7,8,10,11}$. PRDM14 also has a significant role in preimplantation development ${ }^{12}$, as well as pluripotency induction and maintenance in both mouse and human ${ }^{13-16}$. Indeed, PRDM14 knockdown in hESCs led to a decrease in OCT4 levels and elevated expression of lineage markers ${ }^{13,17,18}$.

Despite its critical function in mPGC specification, the role of PRDM14 in hPGC development remains uncertain, due to its low and potentially cytoplasmic expression in gonadal hPGCs ${ }^{3}$. Furthermore, a partial PRDM14 knockdown suggested it might not be important for hPGC specification in vitro ${ }^{19}$, within the TF network for hPGC specification that has diverged significantly from mouse ${ }^{1,6,20}$. In particular, SOX17 is a key determinant of hPGC fate, acting upstream of BLIMP1 and TFAP2C $\mathrm{C}^{3}$, but it is dispensable for mPGC development ${ }^{21,22}$. Understanding whether PRDM14 has a role in hPGC specification is critical towards gaining insights on the molecular divergence between mouse and human PGCs.

An inducible system for PRDM14 loss of function during hPGCLC specification from hESCs is critical, since PRDM14 is also vital for hESC pluripotency ${ }^{13}$. Accordingly, we combined auxin- or jasmonate-inducible degrons ${ }^{23,24}$ with CRISPR/Cas9 genome editing 25 to achieve fast, comprehensive and reversible loss of endogenous PRDM14 protein. We reveal an indispensable role for PRDM14 in germ cell fate, since loss of function affects the efficiency of specification and results in an aberrant hPGCLC transcriptome. Notably, PRDM14 targets are not conserved between mouse and human, reflecting the evolutionary divergence in the molecular network for PGC specification. The study also illustrates the power of conditional degrons, which can be widely used to study TFs during cell fate determination.

\section{Results}

Detection of PRDM14 expression during hPGCLC specification. To follow PRDM14 expression during hPGCLC specification, we appended Venus fluorescent protein to the C-terminus of endogenous PRDM14 (Fig. 1a) in the background of NANOS3tdTomato hPGCLC-specific reporter ${ }^{5}$. PRDM14-T2A-Venus line served for flow cytometry and fluorescence-activated cell sorting (FACS) of PRDM14 ${ }^{+}$cells (Fig. 1b, c), while the fusion PRDM14AID-Venus reporter was used to confirm subcellular localisation of PRDM14 (Fig. 1e), as well as for inducible protein degradation (see below). We detected Venus fluorescence in targeted hESCs and hPGCLCs but not in the parental control (Fig. 1b, c). Immunofluorescence (IF) confirmed co-localisation of Venus and PRDM14 in nuclei of both hESCs and hPGCLCs (Figs. 1e, 2a). Importantly, the majority of alkaline phosphatase (AP) ${ }^{+}$NANOS3-tdTomato $^{+}$ hPGCLCs were PRDM14-Venus ${ }^{+}$(Fig. 1c) and Venus ${ }^{+} \mathrm{AP}^{+}$cells specifically expressed key germ cell markers (Fig. 1d).

We also assessed PRDM14 expression in human gonadal PGCs, and unlike some previous reports ${ }^{3,5}$, we detected PRDM14 not only in the cytoplasm, but also in the nucleus of both male and female Wk7-9 hPGCs, (Supplementary Fig. 1A-D). Indeed, some gonadal sections showed only nuclear PRDM14 (Supplementary Fig. 1A, B). Nuclear localisation was also validated using IF on FACS-purified $\mathrm{AP}^{+}{ }^{+} \mathrm{CKIT}^{+}$gonadal hPGCs (Supplementary Fig. 1E). Based on the available data, PRDM14 localisation is unlikely to be a consequence of stage or sex of hPGCs. However, many more human foetal samples and stages will need to be tested in the future to determine the dynamics, if any, of PRDM14 localisation.

Next, we tracked PRDM14 expression dynamics upon hPGCLC induction by time-course IF, using SOX17 (Fig. 2 and Supplementary Fig. 2A), BLIMP1 (Supplementary Fig. 2B-D), TFAP2C (Supplementary Fig. 2F) and OCT4 (Supplementary Fig. 2A, B) to mark hPGCLCs. PRDM14 was uniformly expressed in 4i hESCs (Fig. 1e) that are competent for hPGCLC fate ${ }^{3}$. However, $12 \mathrm{~h}$ from the start of hPGCLC induction by cytokines, the levels of PRDM14 declined significantly in SOX17 ${ }^{+}$or BLIMP1 ${ }^{+}$cells (Fig. 2a), while the remaining PRDM14-Venus ${ }^{+}$cells at $12 \mathrm{~h}$ were $\mathrm{SOX}^{+}$, and thus likely represented neighbouring pluripotent cells (Supplementary Fig. 2E). The repression of PRDM14 in the putative hPGCLCs was however transient, since we observed specific re-expression of PRDM14 in $\sim 25 \%$ of SOX17 ${ }^{+}$cells on day 1 (D1); the proportion of PRDM $14^{+} \mathrm{SOX} 17^{+}$cells continued to increase progressively, reaching $\sim 60 \%$ on $\mathrm{D} 2, \sim 86 \%$ on $\mathrm{D} 3$, $\sim 91 \%$ on D4 and $\sim 93 \%$ on D5 (Fig. 2b). Many of the remaining SOX17 ${ }^{+}$PRDM14 $4^{-}$or BLIMP1 ${ }^{+}$PRDM14 ${ }^{-}$cells might belong to alternative lineages, as they lacked OCT4 expression (Supplementary Fig. 2A, B). Indeed, PRDM14 marked the majority of BLIMP $1^{+} \mathrm{OCT} 4^{+} / \mathrm{SOX} 17^{+} \mathrm{OCT} 4^{+}$cells in D3-D5 embryoid bodies (Fig. 2c and Supplementary Fig. 2D).

Interestingly, OCT4, while specific to germ cells from D3 onwards, was detected in most cells of the EB at $12 \mathrm{~h}$ and on D1, as well as in many BLIMP1-/SOX17- cells on D2 (Supplementary Fig. 2A, B and ref. ${ }^{3}$ ). Of note, high SOX2 and PRDM14 levels persisted in the control cell aggregates in the absence of hPGCLC-inducing cytokines (Supplementary Fig. 2E).

We also assessed relative expression dynamics of PRDM14 and KLF4, a naive pluripotency-related TF known to be specifically expressed in hPGCs and hPGCLCs ${ }^{3,26}$, but repressed in mouse germ cells ${ }^{27}$. Notably, KLF4 was not expressed in hPGCLC until D3, and KLF4 expression followed that of PRDM14 (Supplementary Fig. 2G).

Importantly, on $\mathrm{D} 2$, a higher proportion of BLIMP1 ${ }^{+}$than of SOX17 ${ }^{+}$cells expressed PRDM14 (Fig. $2 \mathrm{~b}$ and Supplementary Fig. 2C), which agrees with the established order of SOX17 followed by BLIMP1 expression in hPGCLCs ${ }^{3}$. The majority of BLIMP $1^{+}$cells are $\mathrm{SOX}_{17}{ }^{+}$and are therefore likely to progress to the upregulation of PRDM14. By contrast, some SOX17 ${ }^{+}$cells might be at an earlier stage lacking BLIMP1 expression and thus unlikely to have advanced far enough to express PRDM14.

The transient loss followed by re-expression of PRDM14 in cells undergoing hPGCLC specification suggests that PRDM14 might have a vital role in this cell fate decision. We pursued this possibility by using inducible degrons for an acute destabilisation of the PRDM14 protein.

Inducible degrons allow efficient PRDM14 protein depletion. Most inducible loss-of-function approaches act at the transcriptional or post-transcriptional levels and often suffer from slow and incomplete protein removal2 ${ }^{28-31}$. Since PRDM14 is one of the core pluripotency factors in hESCs ${ }^{13}$, and exhibits dynamic changes during hPGCLC induction, conventional knockout approaches are unsuitable to study its functions in hPGCLCs. As PRDM14 expression commences within $24 \mathrm{~h}$ of hPGCLC induction, we decided to use conditional degrons $s^{32}$ to achieve 


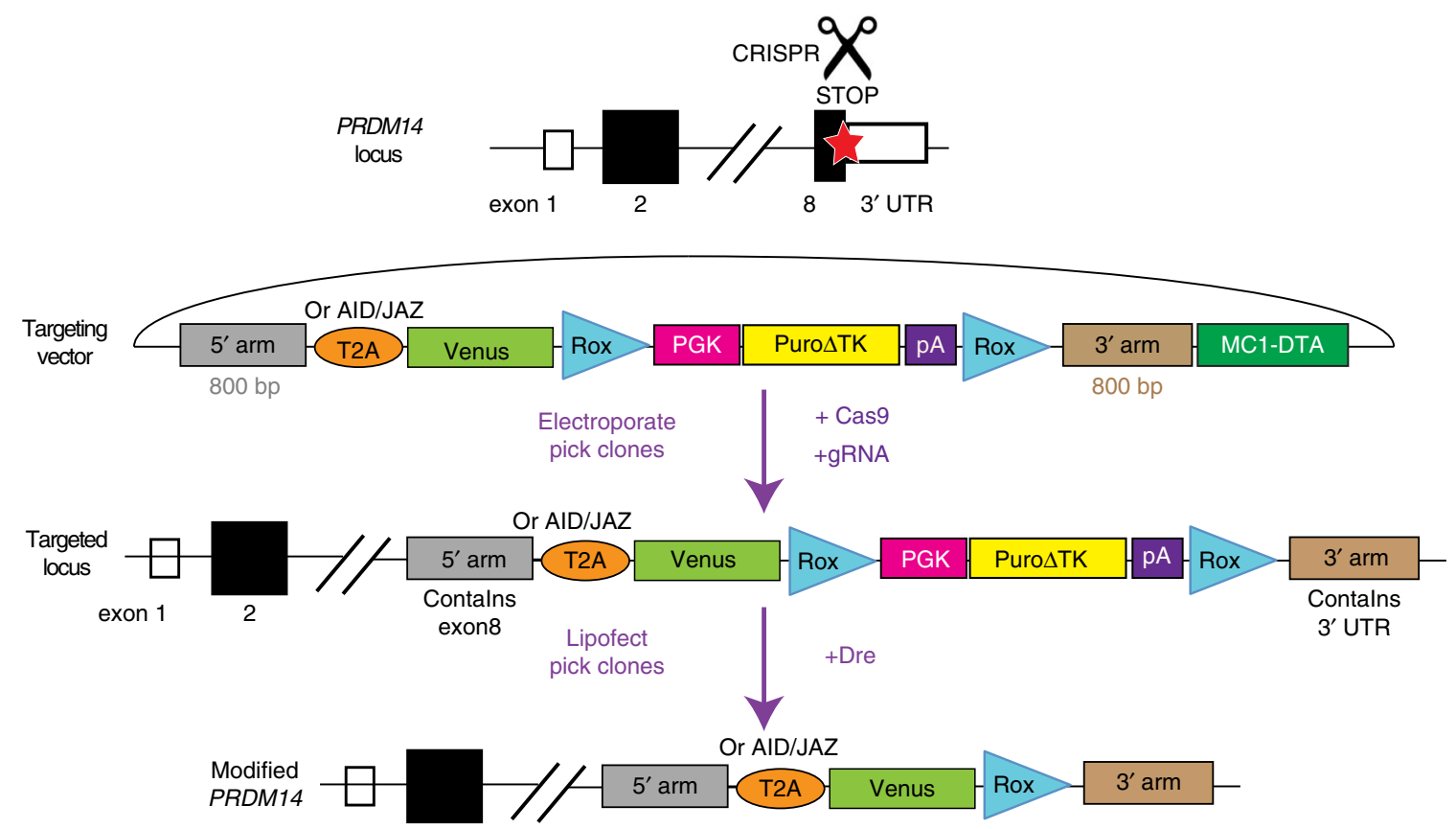

b

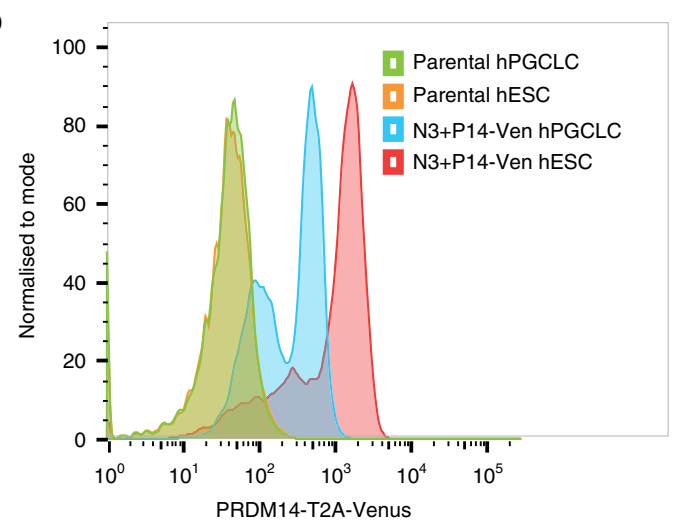

d

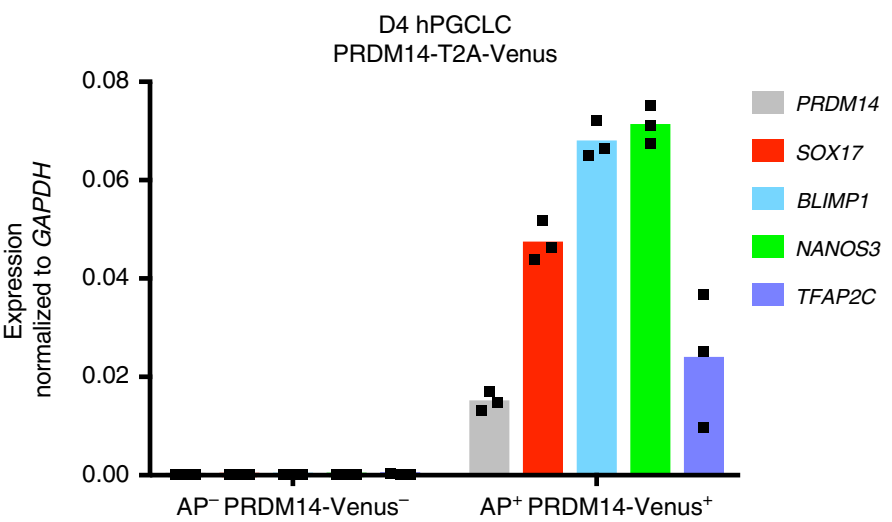

C

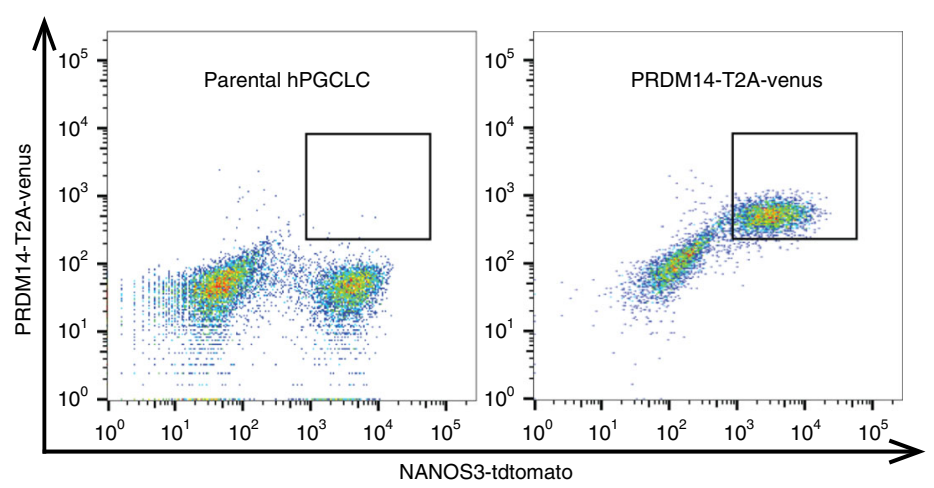

e

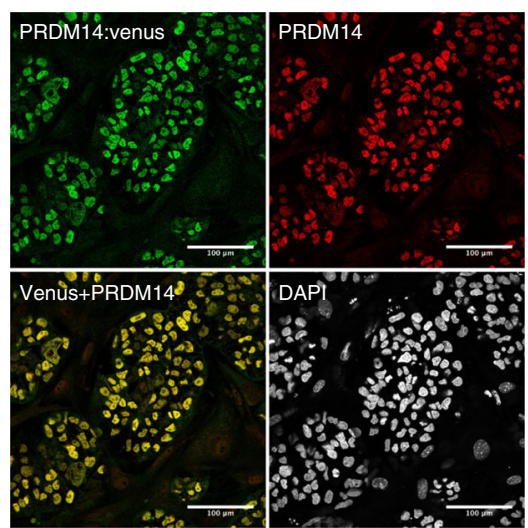

Fig. 1 PRDM14-Venus knock-in reporters allow PRDM14 detection in hESCs and hPGCLCs. a Scheme of CRISPR/Cas9-mediated PRDM14 locus targeting to generate T2A-Venus, AID-Venus or JAZ-Venus reporter versions. $5^{\prime}$ and $3^{\prime}$ arms-homology sequences, T2A-self-cleaving peptide, AID-auxininducible degron, Venus-fluorescent gene, Rox-sequences for site-specific recombination recognised by the Dre enzyme, PGK-Puro-puromycin resistance gene under the control of PGK promoter, $\triangle T K$-truncated thymidine kinase gene, MC1-DTA-diphtheria toxin fragment A gene under the control of MC1 promoter. Also see Fig. 3a. b, c Flow cytometry analysis showing Venus fluorescence in targeted hESCs and hPGCLCs compared with negative control. Note that Venus fluorescence predominantly coincides with NANOS3-tdTomato signal, which marks hPGCLCs. d qPCR analysis on sorted PRDM14-T2A-Venus ${ }^{+} \mathrm{AP}^{+}$and double-negative cells from D4 EBs. Venus ${ }^{+} \mathrm{AP}^{+}$population shows specific expression of germ cell markers. Data show results from three technical replicates (also see Source Data file). e IF analysis (representative of $>10$ experiments) of PRDM14-AID-Venus in competent hESCs showing co-localisation of PRDM14 and Venus fluorescence. Nuclei were counterstained by DAPI. Scale bar is $100 \mu \mathrm{m}$. 
a
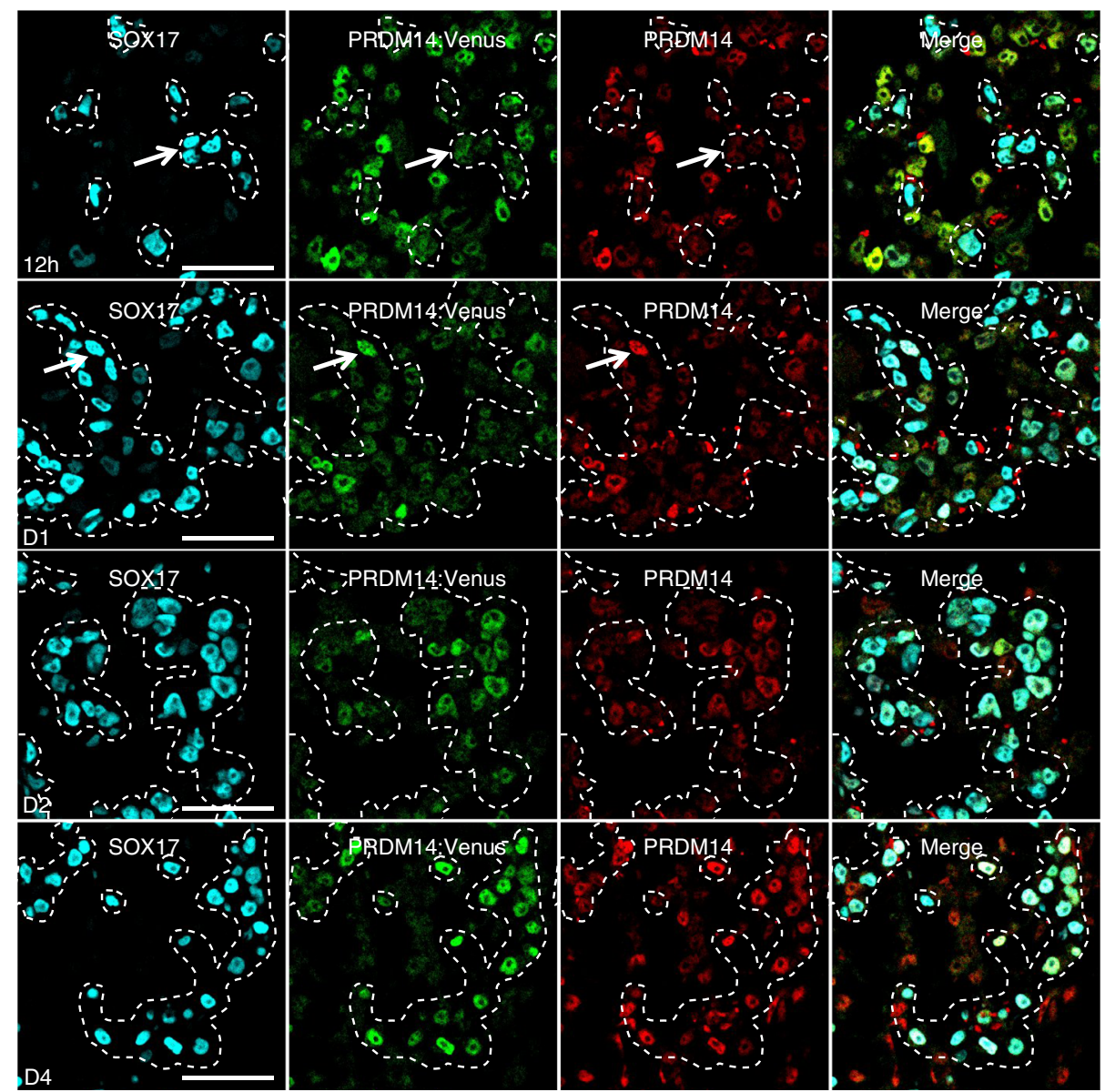

b

PRDM14 expression in SOX $17^{+}$cells

C
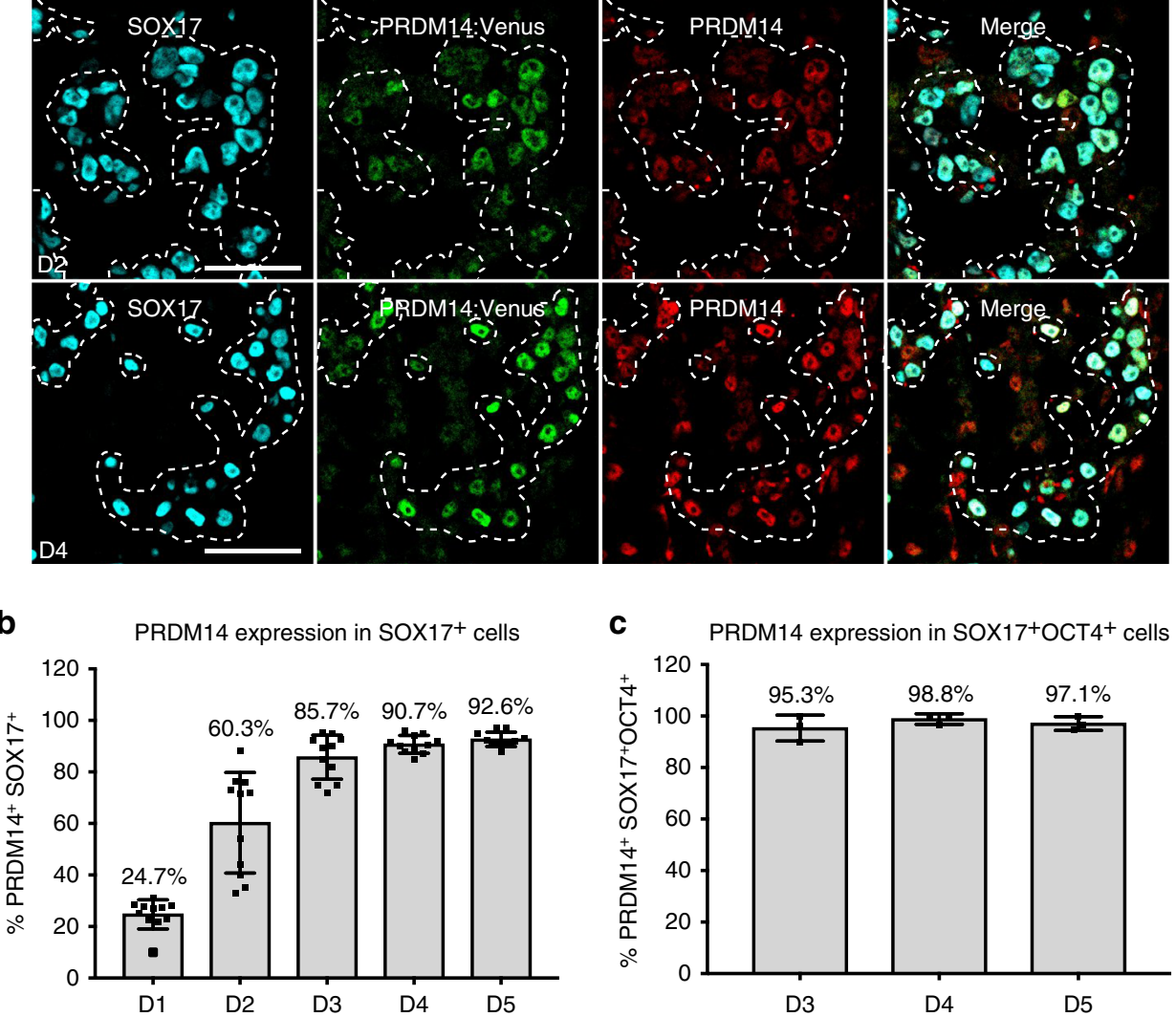

Fig. 2 PRDM14-Venus knock-in reporter detects the dynamics of specific PRDM14 expression in hPGCLCs. a Time-course IF analysis (representative of two hPGCLC inductions) showing PRDM14 expression in embryoid body (EB) sections throughout hPGCLC specification from PRDM14-AID-Venus fusion reporter cell line. hPGCLCs were induced by cytokines and EBs were collected at $12 \mathrm{~h}$, and on D1-D5. Representative images for $12 \mathrm{~h}, \mathrm{D} 1, \mathrm{D} 2$ and D4 are shown. hPGCLCs were marked by SOX17 and highlighted by a dashed line. Arrows show examples of SOX17+ cells that are PRDM14-negative at $12 \mathrm{~h}$ and PRDM14-positive on D1 of differentiation. Scale bar is $60 \mu \mathrm{m}$. b Quantification of results from a and Supplementary Fig. 2A, including the D1-D5 time

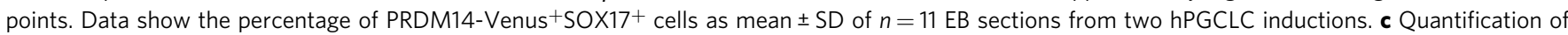

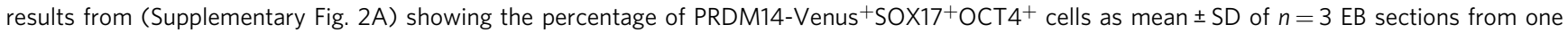
hPGCLC induction.

fast, inducible and reversible PRDM14 depletion at the protein level.

Auxin and jasmonate-inducible degrons (AID and JAZ, respectively), are plant pathways for ligand-induced targeted protein degradation ${ }^{23,24}$. To harness AID and JAZ degrons in hESCs and hPGCLCs, we used CRISPR/Cas9 to tag the Cterminus of the endogenous PRDM14 with AID or JAZ degron sequences (see below) fused to Venus (Fig. 3a). Next, we employed the PiggyBac transposon system ${ }^{33}$ to deliver codonoptimised transgenes encoding cognate hormone receptors from rice (Oryza sativa): TIR1 for AID and COI1B for JAZ, allowing target degradation upon administration of auxin (indole-acetic acid, IAA) or coronatine (Cor), respectively ${ }^{24,34}$. Note that we fused COI1B with TIR1 F-box domain (resulting in Fb-TIR1COI1B) to ensure better integration into human SCF (SKP1, CUL1 and F-box) E3 ubiquitin-ligase complex, as reported previously ${ }^{24}$.

For AID, we chose the 44-amino-acid (residues 71-114) version of the Arabidopsis thaliana AID tag $\left(\right.$ AtAID $\left.^{44}\right)$ that was used successfully elsewhere ${ }^{34,35}$. Since the JAZ system has not 
a
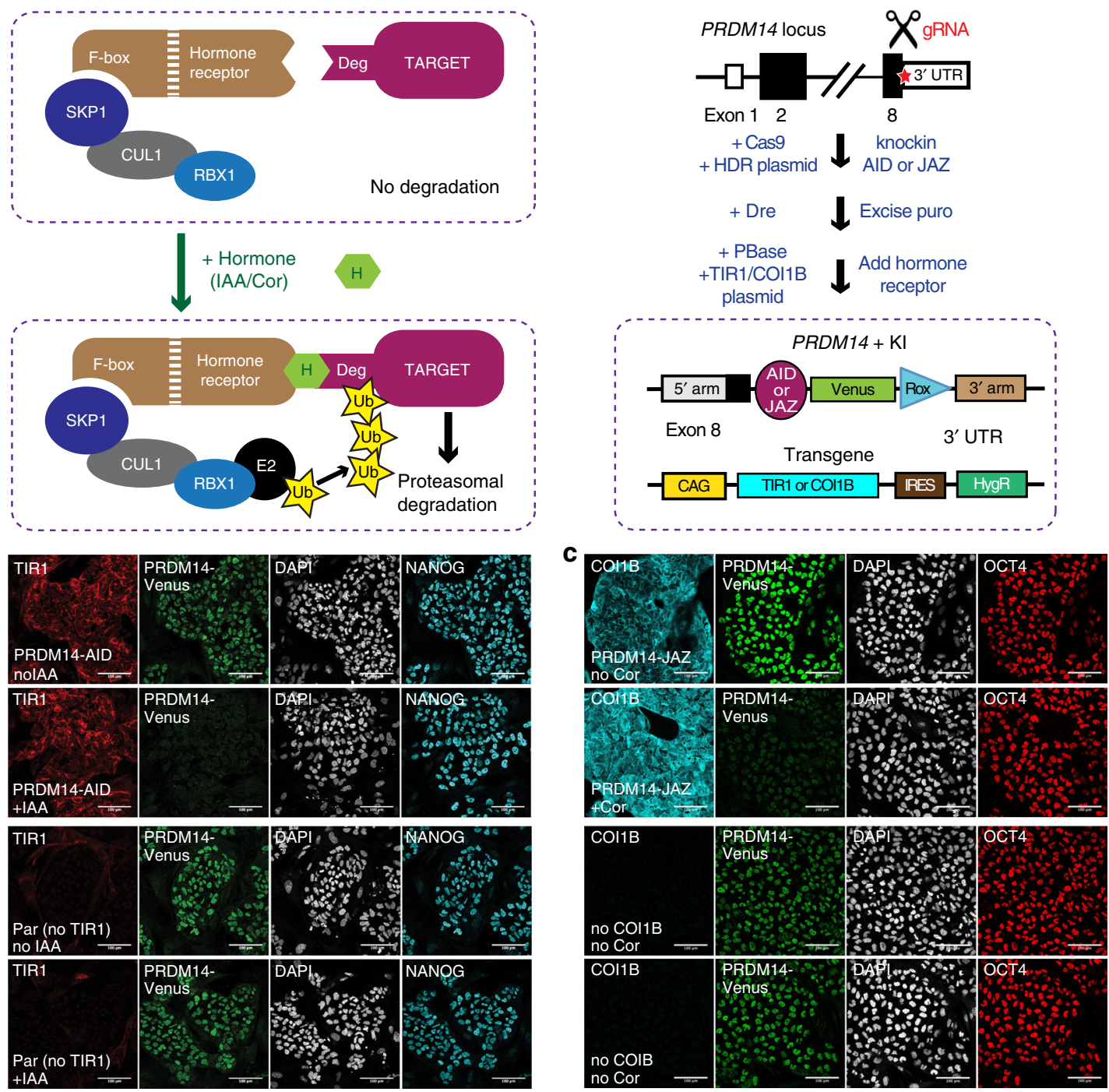

Fig. 3 Auxin- and jasmonate-inducible degrons allow fast and homogenous PRDM14 protein depletion. a Scheme of AID and JAZ systems and the derivation of PRDM14-AID-Venus and PRDM14-JAZ-Venus cell lines. SKP1, CUL1, RBX1 and F-box (TIR1 or COI1B) are the subunits of the SCF E3 ubiquitin-ligase complex. In the presence of IAA (for AID) or Cor (for JAZ) the target associates with the E3 ligase via a cognate hormone receptor (F-box protein); the recruited E2 ubiquitin-conjugating enzyme polyubiquitinates the target, which results in its proteasomal degradation 24,80 . For cell line generation, the cells were first transfected with two plasmids: (1) Cas9-encoding plasmid 25 with a gRNA sequence targeting the vicinity of the PRDM14 stop codon; (2) HDR (homology-directed repair) template (also see Fig. 1a) containing the sequences to be appended by Kl, flanked by two 800-bp homology arms; MC1-DTA was added to reduce random integration. The knocked-in sequences were: AID (AtAID ${ }^{44}$ ) or JAZ (OsJAZ43) followed by Venus and a positive/negative PGK-puromycin- $\Delta$ TK selection cassette flanked by Rox sites to allow excision by Dre ${ }^{5}$. Correct homozygous clones were transfected with PiggyBac constructs encoding the corresponding hormone receptor (TIR1 for AID and Fb-TIR1-COI1B for JAZ) under the control of the CAG promoter followed by an IRES and a hygromycin resistance gene. The homogeneity of myc-TIR1 or HA-COI1B expression in selected clones was checked by IF (see b, c). b IF (representative of three experiments) showing PRDM14, NANOG and TIR1 (myc-tagged) expression and PRDM14-AID-Venus depletion upon $25 \mathrm{~min}$ of IAA treatment. Nuclei were counterstained by DAPI. Scale bar is $100 \mu \mathrm{m}$. c IF (representative of three experiments) showing PRDM14-Venus, OCT4 and COI1B (HA-tagged) expression and PRDM14-JAZ-Venus depletion upon 2 days of Cor treatment. Nuclei were counterstained by DAPI. Scale bar is $100 \mu \mathrm{m}$.

been validated as extensively as AID, we performed additional optimisation experiments (Supplementary Fig. 3). We compared the best performing degron sequences from ${ }^{24}$, namely OsJAZ ${ }^{33}$ (version 6-Os33) and NLS-OsJAZ ${ }^{33}$ (version 7), with a slightly longer OsJAZ ${ }^{43}$ degron sequence (without NLS). For this, HEK293T cells were infected with viral constructs harbouring GFP fused to respective degron sequences and treated with Cor or DMSO (control). GFP depletion at 2, 4 and $24 \mathrm{~h}$ was monitored by flow cytometry (Supplementary Fig. $3 \mathrm{~A}$ ). This showed that the longer degron sequence $\left(\mathrm{OsJ} \mathrm{AZ}^{43}\right)$ is superior to $O s \mathrm{JAZ}^{33}$ and slightly better than NLS-OsJAZ ${ }^{33}$ in the efficiency and speed of GFP depletion (Supplementary Fig. 3A). This suggests that OsJAZ $\mathrm{Z}^{43}$ is more versatile and can allow depletion of both nuclear and cytoplasmic proteins.

To further test if JAZ could be efficiently used in our system, we generated hESCs expressing a PiggyBac-delivered construct (Supplementary Fig. 3B), where GFP was fused to the best OsJAZ ${ }^{43}$ degron sequence and an NLS, to better model the depletion of TFs such as PRDM14. The construct also harboured the Fb-TIR1-COI1B hormone receptor. GFP signal started 
decreasing $3 \mathrm{~h}$ after Cor administration (Supplementary Fig. 3C) and $99 \%$ of cells were GFP-negative after one passage in Cor (Supplementary Fig. 3D). Based on these results, we used OsJAZ ${ }^{43}$ and Fb-TIR1-COI1B combination to deplete PRDM14.

First, we tested AID- or JAZ-mediated PRDM14 depletion in hESCs by growing them with or without corresponding hormones and performing IF (Fig. 3b, $\mathrm{c}$ and Supplementary Fig. 4). PRDM14 and Venus fully colocalised and were both reduced upon the addition of IAA or Cor in hormone-sensitive, but not in parental lines (Fig. $3 \mathrm{~b}, \mathrm{c}$ and Supplementary Fig. 4A-D). Crucially, the AID system allowed reduction of Venus fluorescence to negligible levels, comparable with cells lacking the Venus knock-in (Supplementary Fig. 4C). Timecourse IF in hESCs revealed a rapid onset of PRDM14-AIDVenus depletion within $10 \mathrm{~min}$ of IAA treatment, reaching homogeneity within 25 min (Fig. 3b and Supplementary Fig. 4E). In the case of the JAZ degron, however, residual PRDM14-JAZVenus signal was detectable even after 2 days of Cor treatment (Fig. 3c and Supplementary Fig. 4D). Both systems are nevertheless reversible ${ }^{24,34}$, and IAA wash-off restored PRDM14 levels in less than $2 \mathrm{~h}$ (Supplementary Fig. 4F). Altogether, inducible degrons allow fast and reversible PRDM14 depletion, with AID being superior in terms of speed and efficiency.

Inducible PRDM14 degradation reduces hPGCLC specification. Next, we addressed the importance of PRDM14 for hPGCLC specification by adding IAA or Cor at the onset of hPGCLC differentiation (D0), followed by measuring the induction efficiency on D4 by recording the percentage of NANOS3tdTomato ${ }^{+} \mathrm{AP}^{+}$cells (Fig. 4a). Notably, PRDM14 depletion using both approaches resulted in a significant reduction of hPGCLC induction efficiencies (by $70 \%$ and $30-60 \%$, respectively) (Fig. 4b, $c$ and Supplementary Fig. 5A-C). The effect was more pronounced in the case of the AID system, presumably due to its higher efficiency and faster kinetics of PRDM14 depletion. Therefore, we focused on the AID system to study PRDM14 further. Crucially, the observed phenotype was replicated using a PRDM14-AID in another hESC line (Supplementary Fig. 5D).

To further test AID efficacy in this context, we induced degradation of SOX17, a known hPGC and definitive endoderm (DE) regulator ${ }^{3,20,36}$. IAA addition fully abrogated hPGCLC and DE specification (Supplementary Fig. 5E-G), confirming the key roles of SOX17 in these lineages.

To establish the time when PRDM14 is essential for hPGCLC specification, we performed a time-course of IAA supplementation. We performed depletion of PRDM14 by adding IAA starting on D0, D1 or D2, followed by analysis on D4. We noted a strong phenotype with reduction in hPGCLC specification (Fig. 4d). By contrast, the addition of IAA on D3 had no detectable effect on the number of NANOS3-tdTomato ${ }^{+} \mathrm{AP}^{+}$ cells (Fig. 4d). This suggests that either PRDM14 is dispensable after D2 or that its depletion at later time points does not affect hPGCLC numbers, although transcriptional or epigenetic consequences cannot be excluded. Altogether this points to an early critical role of PRDM14 in hPGCLC specification.

PRDM14 is highly expressed in human PGC-competent pluripotent cells, unlike in mice, where it is repressed at the equivalent stage ${ }^{3,37}$. Notably, however, PRDM14 depletion in 4i hESCs for one passage prior to hPGCLC induction (Supplementary Fig. 5H) had no effect on hPGCLC specification (Supplementary Fig. 5I), indicating no detectable involvement of PRDM14 in the maintenance of competence for hPGCLC fate. To determine if PRDM14 is required for the acquisition of competence, we induced competence for hPGCLCs in hESCs via pre-mesendoderm (preME), which transiently displays hPGCLC potential ${ }^{5}$. However, when IAA was added to the preME medium and washed off before hPGCLC induction (Supplementary Fig. 5H), we observed no effect on hPGCLC specification efficiency (Supplementary Fig. 5J). Altogether, these data demonstrate that PRDM14 is important for hPGCLC specification but is dispensable for the acquisition and maintenance of the hPGCLC-competent state. Furthermore, this suggests a probable specific and direct requirement for PRDM14 in hPGCLC induction, which might not simply result from pluripotency disruption in the starting cell population.

Restored PRDM14 levels rescue hPGCLC differentiation. To confirm the specificity of the observed phenotype, we attempted to rescue the endogenous PRDM14 depletion with ectopic PRDM14, using the ProteoTuner system, whose kinetics is comparable with that of $\mathrm{AID}^{38}$. PRDM14 transgene was fused to a destabilisation domain (DD) and thus continuously degraded by the proteasome but stabilised by Shield-1 ligand ${ }^{38,39}$. Interestingly, overexpression of PRDM14 on D0, but not on D1 could fully rescue hPGCLC specification efficiency and even enhanced hPGCLC specification compared with "no IAA" control (Fig. 5a). This suggests that the transient PRDM14 repression observed at the onset of hPGCLC specification (Fig. 2b) is not prerequisite for germ cell induction and that despite low PRDM14 expression in SOX17/BLIMP1 ${ }^{+}$cells on D0, it is apparently critical for hPGCLC induction.

We then asked if we could restore the endogenous PRDM14 levels by blocking auxin perception. To achieve this, we designed an AID-JAZ degron switch, where PRDM14-AID-Venus is degraded by IAA supplementation, while TIR1 is fused to JAZ and thus depleted by Cor (Fig. 5b). IF analysis in hESCs confirmed that simultaneous administration of both IAA and Cor desensitised hESCs to IAA via TIR1 degradation and replenished the PRDM14-Venus pool (Fig. 5c). In line with previous experiments, these cell lines also showed decreased hPGCLC specification efficiency upon IAA treatment (Fig. 5d). Crucially, in the presence of both IAA and Cor, hPGCLC differentiation efficiency was significantly restored (Fig. 5d). Interestingly, similar to PRDM14-DD overexpression above (Fig. 5a), significant rescue was only achieved on D0, but not on D1-D2 (Fig. 5e), confirming the early role of PRDM14 in hPGCLC specification. Overall, these data prove the causative role of PRDM14 depletion in hPGCLC induction phenotype and exemplify the use of orthogonal degron approaches for rescue design.

PRDM14-deficient hPGCLCs show aberrant transcriptome. Next, we examined the transcriptome of PRDM14-deficient hPGCLCs by performing RNA-sequencing (RNA-seq) on NANOS3-tdTomato ${ }^{+} \mathrm{AP}^{+}{ }^{\text {hPGCLCs }}$ differentiated with or without IAA for 4 days from two hormone-sensitive clones and the parental control lacking TIR1. We also addressed the role of PRDM14 in pluripotency regulation, by analysing the transcriptome of $\mathrm{AP}^{+}$competent hESCs grown with or without IAA for one passage (3 days). Importantly, the parental ("no TIR1") control showed no differentially expressed genes (DEGs) in IAAtreated hESCs, and only two upregulated genes in hPGCLCs (CYP1B1 and LRAT), indicating minimal non-specific effects of auxin on the target cells' transcriptome (Supplementary Data 1).

RNA-seq on PRDM14-depleted hESCs identified 106 upregulated and 64 downregulated genes (Fig. 6a and Supplementary Data 1). Crucially, our findings concur with a previous study on PRDM14 in different hESC lines and culture conditions ${ }^{13}$ (Fig. 6c), with downregulation of pluripotency genes (e.g. OCT4, NANOG, TDGF1 and GAL), and upregulation of differentiation 
a
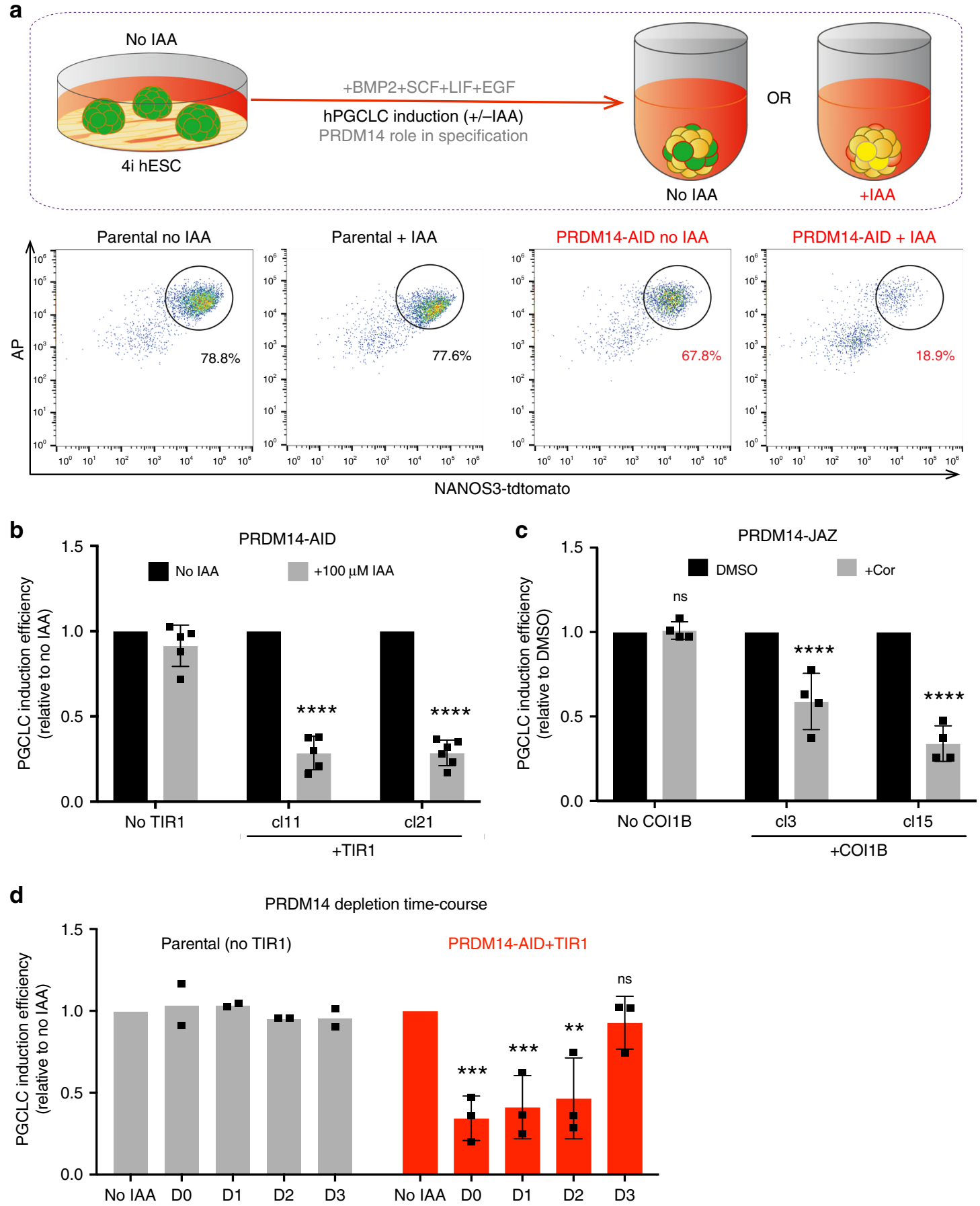

Fig. 4 PRDM14 depletion using AID or JAZ degrons reduces hPGCLC specification efficiency. a Experimental design and representative flow cytometry plots showing reduced hPGCLC induction in hormone-sensitive clones, but not in the parental control. hPGCLCs were defined as NANOS3-tdTomato ${ }^{+}$AP ${ }^{+}$ cells. b Quantification of flow cytometry results for PRDM14-AID-Venus hPGCLCs induced with or without IAA (100 $\mu$ M). The induction efficiency of untreated cells ("no IAA") was set to 1 . Data show mean \pm SD for $n=5$ for cl11 and "no TIR1" control and $n=6$ for cl21, ns not significant $(P=0.1308)$, ${ }^{\star * \star \star} P<0.0001$ (Two-way ANOVA followed by Sidak's multiple comparison test). c Quantification of flow cytometry results for PRDM14-JAZ-Venus hPGCLCs. The induction efficiency of untreated cells ("no Cor") was set to 1 . Data show mean \pm SD for $n=4$ independent experiments per clone, ns not significant $(P=0.9979)$, ${ }^{\star \star \star \star} P<0.0001$ (Two-way ANOVA followed by Sidak's multiple comparison test). d Time-course of PRDM14 depletion. IAA (500 $\mu \mathrm{M}$ ) was added on indicated days of hPGCLC induction from PRDM14-AID-Venus clones. Note that IAA was not washed off until the end of differentiation (D4), when hPGCLC induction efficiency was assessed by flow cytometry. Data show mean \pm SD of $n=3$ for PRDM14-AID or mean of $n=2$ for "no TIR1" control. Statistical significance was analysed by two-way ANOVA followed by Sidak's multiple comparison test. For PRDM14-AID: no IAA vs. D0 *** $(P=$ $0.0003)$, no IAA vs. D1 ${ }^{\star \star \star}(P=0.0008)$, no IAA vs. D2 ${ }^{\star \star}(P=0.0018)$, no IAA vs. D3 ns (not significant, $\left.P=0.9614\right)$; for "no TIR1": all ns not significant $(P>0.99)$ 
a

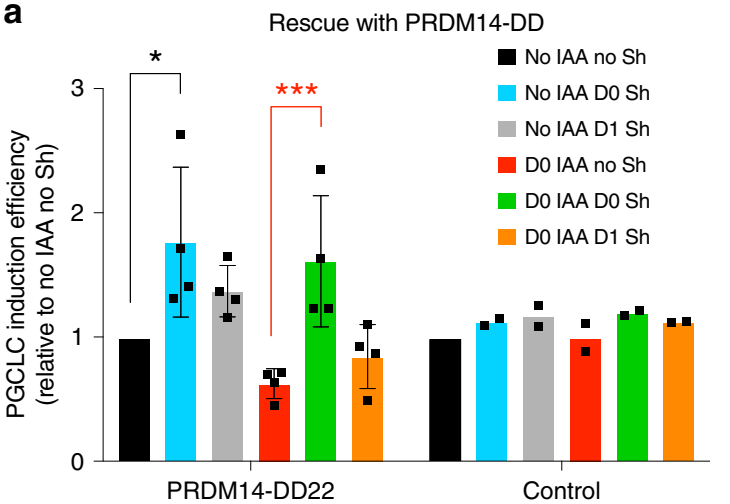

b

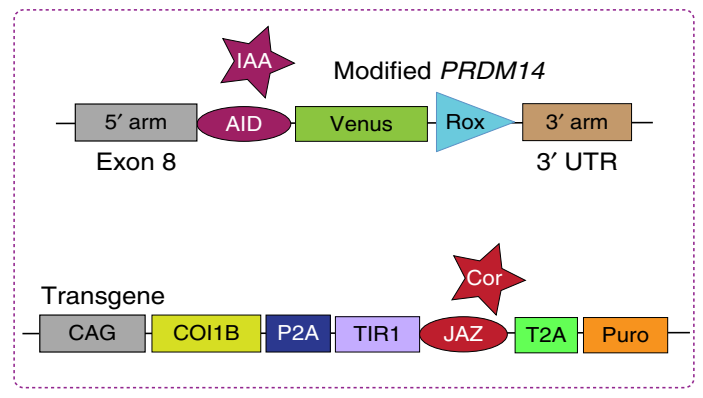

C

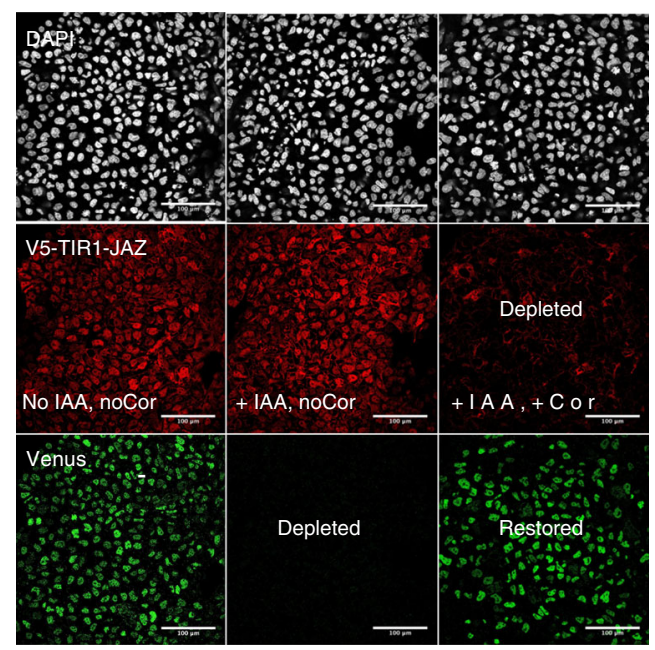

e

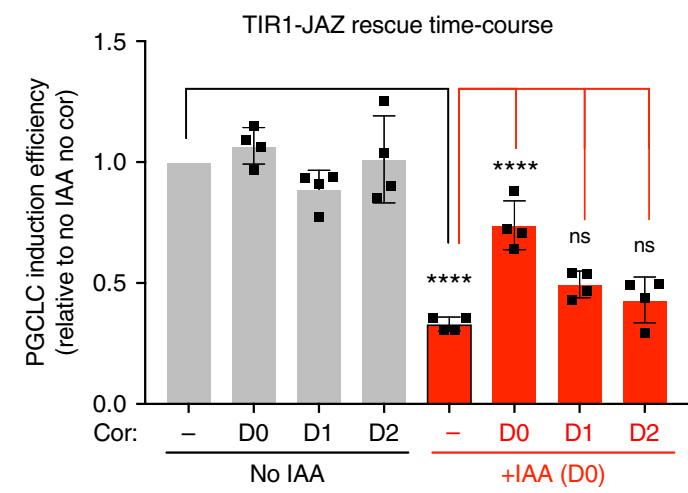

d

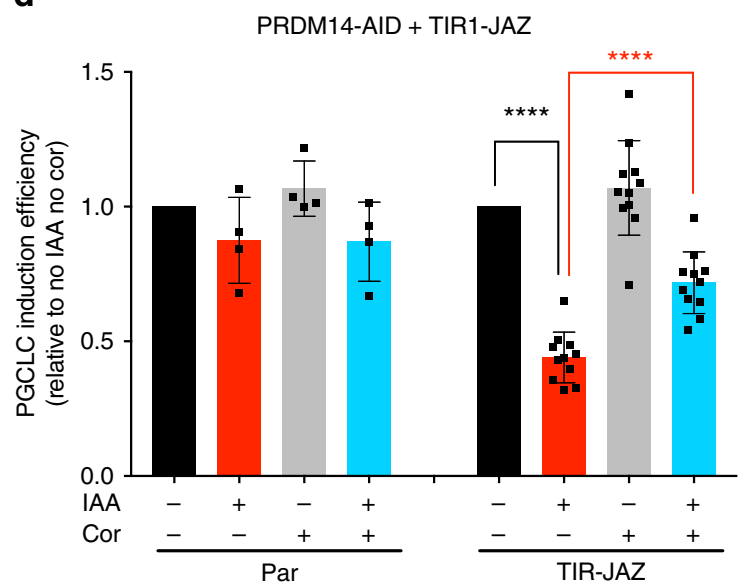

Fig. 5 Ectopic PRDM14 expression or desensitisation to auxin rescue hPGCLC specification. a IAA and/or Shield-1 (Sh) were added on indicated days of differentiation to deplete the endogenous PRDM14 or stabilise the PRDM14-DD transgene, respectively. "Control" cell line lacks both the AID knock-in and the PRDM14-DD transgene. Data show mean \pm SD for $n=4$ (PRDM14-DD22) or mean of $n=2$ (control) independent experiments. Statistical significance was analysed by two-way ANOVA followed by Sidak's multiple comparison test; for PRDM14-DD22: D0 IAA no Sh vs. no IAA no Sh not significant, ( $P=$ $0.4159)$, DO IAA no Sh vs. no IAA DO Sh ${ }^{\star \star \star}(P=0.0001)$, DO IAA no Sh vs. no IAA D1 Sh ${ }^{\star}(P=0.0125)$, DO IAA no Sh vs. DO IAA DO Sh ${ }^{\star \star *}(P=$ $0.0008)$, D0 IAA no Sh vs. D0 IAA D1 Sh not significant $(P=0.8662)$; for control: all not significant $(P>0.97)$. b Scheme of the double AID/JAZ degron design. Endogenous PRDM14 is under the control of the AID degron as above, while the stability of TIR1 hormone receptor is controllable by the JAZ degron. c AID/JAZ validation by IF in hESCs (representative of three experiments). Simultaneous IAA and Cor supplementation depletes TIR1 and restores PRDM14, as shown by IF staining for PRDM14-AID-Venus and TIR1(V5-tagged). Nuclei were counterstained by DAPI. Scale bar is $100 \mu \mathrm{m}$. d Desensitisation to auxin via the AID/JAZ degron switch rescues hPGCLC induction efficiency. Data show mean \pm SD for $n=4$ (no TIR1 control) or $n=11$ (TIR-JAZ) biological replicates, ${ }^{\star \star \star \star} P<0.0001$ (Two-way ANOVA followed by Sidak's multiple comparison test). e Time-course of desensitisation to auxin via the AID/JAZ degron switch. Note that hPGCLC induction efficiency is only significantly rescued if Cor is supplemented from DO. Addition of Cor alone (without IAA) does not alter hPGCLC specification efficiency. Data show mean \pm SD for $n=4$ biological replicates, ns not significant (D0 IAA no Cor vs. D0 IAA D1 Cor $P=0.3982$; D0 IAA no Cor vs. D0 IAA D2 Cor $P=0.9832$ ), ${ }^{\star \star \star \star} P<0.0001$ (Two-way ANOVA followed by Sidak's multiple comparison test). 
a
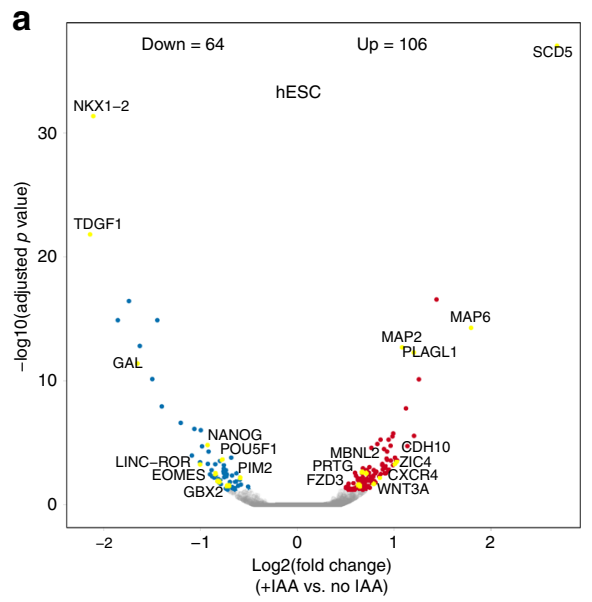

C

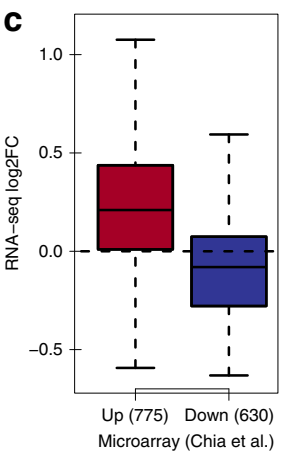

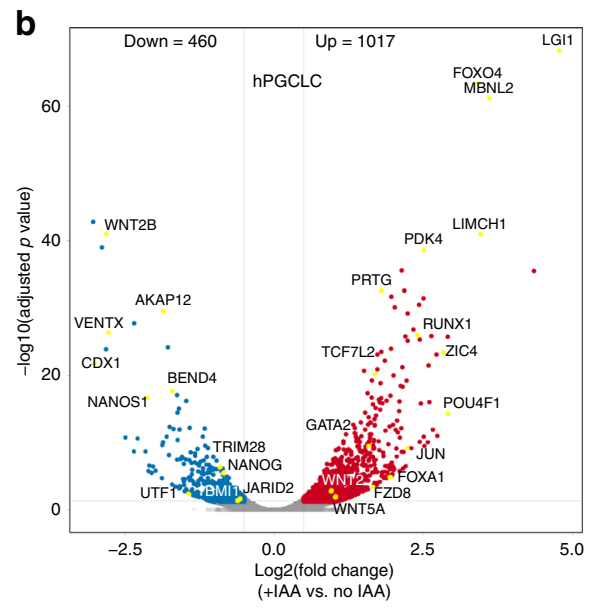

d

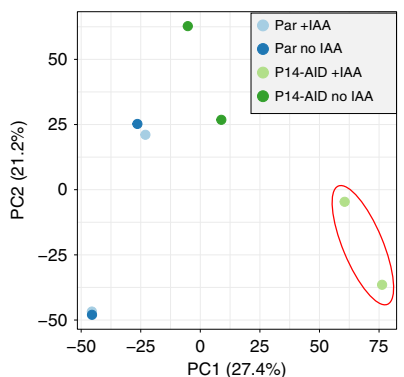

Soma no IAA Soma +IAA

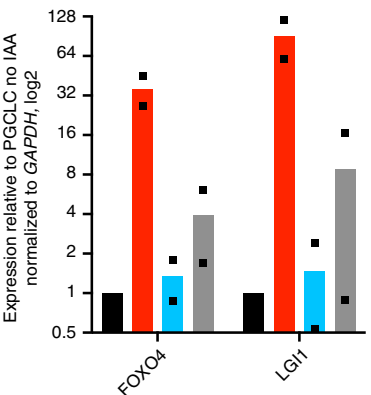

f
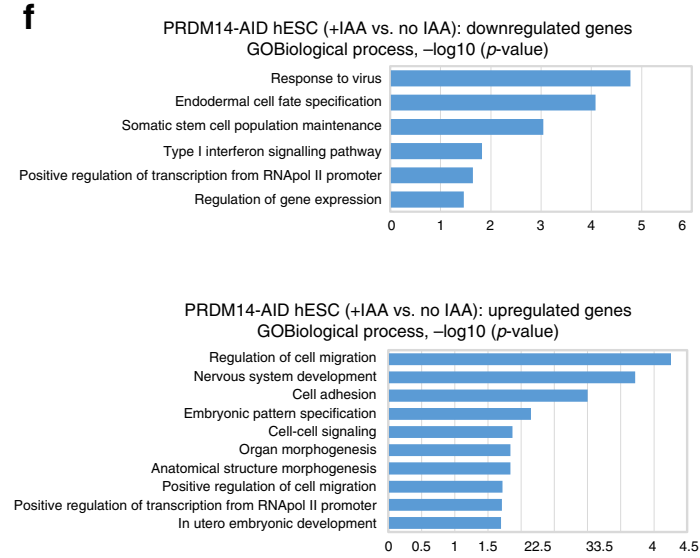

markers, particularly of the neural lineage (e.g. MAP2, MAP6 and SOX2) (Fig. 6a, Supplementary Fig. 6E and Supplementary Data 1). Gene ontology (GO) analysis reflected reduction in self-renewal (enriched downregulated term "somatic stem cell maintenance") and upregulation of pro-differentiation genes

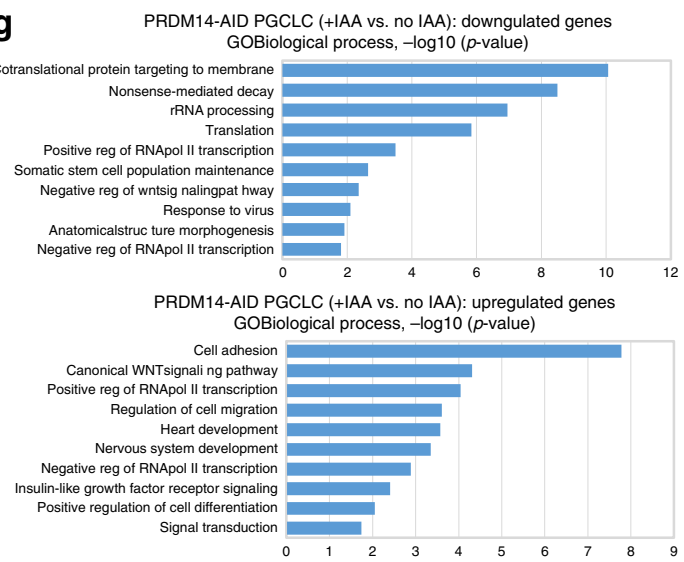

(enriched terms "nervous system development" and "embryonic pattern specification") (Fig. 6f).

Notably, there was a stronger response to PRDM14 loss in hPGCLCs, with 1017 upregulated and 460 downregulated genes in "+IAA" vs "no IAA" samples (Fig. 6b), and only a small 
Fig. 6 RNA-seq reveals transcriptional changes in PRDM14-deficient hESCs and hPGCLCs. a Volcano plot showing down- $(\log 2$ FC $<(-0.5)$, padj $<$ 0.05) and upregulated $(\log 2 \mathrm{FC}>0.5$, padj $<0.05)$ genes in hPGC-competent hESCs upon PRDM14 loss. PRDM14-AID hESCs were treated with IAA for one passage ( 3 days) and sorted as AP+ cells for RNA-seq along with "no IAA" control. b Volcano plot showing down- $(\log 2 \mathrm{FC}<(-0.5)$, padj $<0.05)$ and upregulated $(\log 2 \mathrm{FC}>0.5$, padj $<0.05)$ genes in hPGCLCs upon PRDM14 loss. PRDM14-AID hPGCLCs were specified with or without IAA for 4 days and sorted as NANOS3-tdTomato ${ }^{+} \mathrm{AP}^{+}$cells for RNA-seq along with "no IAA" control. c Boxplot overlaying down- $(\log 2 \mathrm{FC}<(-0.5)$, padj $<0.05)$ and upregulated $(\log 2 \mathrm{FC}>0.5$, padj $<0.05)$ genes upon PRDM14 knockdown in conventional hESCs (microarray data from ${ }^{13}$ ) and RNA-seq analysis in PRDM14-AID-depleted PGC-competent (4i) hESCs from this study. The distance from the bottom to the upper line of the box spans the interquartile range $(I Q R)$, the horizontal line in the box indicates the median of the dataset, while the whiskers show $\pm 1.5 \times I Q R$. Note that the majority of differentially expressed genes show consistent changes. d Principal component analysis (PCA) of hPGCLC RNA-seq samples. PRDM14-depleted hPGCLCs (cl11 and cl21; highlighted in red) separate from the other samples along principal component 1 (PC1). e qPCR validation of selected DEGs from RNA-seq. hPGCLCs from cl11 and cl21 were sorted as NANOS3-tdTomato ${ }^{+} \mathrm{AP}^{+}$cells, while soma denotes the double-negative population from the same experiments. Strongly upregulated genes are plotted separately. Data show gene expression relative to no IAA hPGCLCs and normalised to GAPDH, mean \pm SEM of $n=5$ for SOX17 and VENTX, mean \pm SEM of $n=3$ independent experiments for NANOS3, BLIMP1, TFAP2C, KLF4 or $n=2$ for other genes. Statistical significance of hPGCLC no IAA vs. +IAA was assessed by multiple unpaired $t$-tests yielding two-tailed $P$ values: $K L F 4{ }^{\star *}(P=0.0054), N A N O G{ }^{* *}(P=0.0053), N A N O S 3$ ${ }^{\star}(P=0.0452)$, VENTX ${ }^{\star \star \star *}(P<0.0001)$. The parental ("no TIR1") control is shown in Supplementary Fig. 6D. f Gene ontology (GO) analysis on down- and upregulated genes in PRDM14-deficient hESCs. Top non-redundant GO terms are shown as $-\log 10(p$ value). $\mathbf{g}$ GO analysis on down- and upregulated genes in PRDM14-deficient hPGCLCs. Top non-redundant GO terms are shown as $-\log 10$ ( $p$ value). For complete list of GO terms see Supplementary Data 6.

overlap between the affected genes in hESCs and hPGCLCs (Supplementary Data 1). Principal component analysis (PCA) and unsupervised hierarchical clustering of hPGCLC transcriptome confirmed that parental control, irrespective of IAA treatment, clustered with untreated hormone-sensitive clones, and away from the PRDM14-depleted hPGCLCs that clustered together (Fig. 6d and Supplementary Fig. 6A). Crucially, PRDM14-deficient hPGCLCs showed downregulation of many key hPGC- and pluripotency-related genes, including UTF1, NANOG, NANOS1, LIN28A and TRIM28. GO analysis showed enrichment of protein biosynthesis-related processes, somatic stem cell population maintenance and negative regulation of WNT signalling (Fig. 6h). Gene set enrichment analysis (GSEA) against 123 core PGC genes defined previously ${ }^{5}$, showed lower enrichment score of mutant hPGCLCs compared with the control (Supplementary Fig. 6B). The levels of key hPGC regulators, SOX17 and PRDM1, however did not change (Fig. 6e and Supplementary Fig. 6D), which is consistent with their upregulation prior to PRDM14 in nascent hPGCLCs (Fig. 2, Supplementary Fig. 2A, B and ref. ${ }^{3}$ ). Crucially, top changes in gene expression were also confirmed by $\mathrm{qPCR}$ using independent hPGCLC inductions (Fig. 6e and Supplementary Fig. 6D). qPCR analysis also showed downregulation of KLF4 in PRDM14depleted hPGCLCs (Fig. 6e), which, together with the observation that PRDM14 expression precedes that of KLF4 (Supplementary Fig. 2G), might suggest that PRDM14 could be upstream of KLF4 in hPGCLCs. Ectopic PRDM14 could partially revert the transcriptional changes in mutant hPGCLCs (Supplementary Fig. 6C), in line with rescued hPGCLC specification phenotype (Fig. 5a).

GO analysis on genes derepressed in PRDM14-deficient hPGCLCs identified enrichment of terms related to WNT signalling, as well as heart and nervous system development (Fig. 6g). A similar response occurs in hPGCLCs specified without BLIMP1 or TFAP2C 20,26 . Indeed, pairwise comparisons revealed a significant overlap between both up- and downregulated targets, suggesting potential combinatorial roles of PRDM14 with BLIMP1 and TFAP2C. Accordingly, 115 genes were downregulated in all three mutants (including germ cell markers UTF1, NANOG, AKAP12, NANOS1 and TRIM28), while 281 shared upregulated genes were mostly implicated in morphogenesis, WNT signalling, as well as cell migration and adhesion (Supplementary Fig. 6F-G and Supplementary Data 2).

PRDM14 regulates gene expression through promoter binding. To identify direct targets of PRDM14 in hPGCLCs and hESCs, we performed chromatin immunoprecipitation (ChIP) with highthroughput sequencing (ChIP-seq). We took advantage of the PRDM14-AID-Venus line to use a ChIP-grade anti-GFP antibody for PRDM14-Venus immunoprecipitation, which proved more efficient than anti-PRDM14 antibodies (Supplementary Fig. 7A). Importantly, auxin treatment led to a significant loss of signal (Supplementary Fig. 7A), which allowed us to use ChIP-seq on IAA-treated hESCs as a control.

Overall, ChIP-seq identified 6486 consensus PRDM14 peaks: 4206 in hPGCLCs and 3319 in hESCs (Supplementary Data 3). $k$ means clustering partitioned the dataset into five distinct clusters (Fig. 7a), separating peaks specific to hESCs (742 peaks, cluster 4) or hPGCLCs (1331 peaks, cluster 5) or shared between the two (clusters 1-3). Note that the strongest ChIP-seq enrichment persisted in IAA-treated hESCs (clusters 1-2) with 1728/3319 hESC binding sites detectable in hESC + IAA, albeit with significantly lower signal (Fig. 7a and Supplementary Data 3). Such persistent regions might be more tightly bound, and/or require longer auxin exposure to eliminate PRDM14 binding completely.

Notably, PRDM14 in both hESCs and hPGCLCs predominantly binds within $1 \mathrm{~kb}$ of transcription start sites (TSS) (Supplementary Data 3 ), with $\sim 30 \%$ of peaks spanning annotated promoters (Fig. 7b), which agrees with a previous ChIP-seq in hESCs $^{13}$. By contrast, PRDM14 binds predominantly to distal genomic regions in mice, and only $4-10 \%$ of peaks are located within $1 \mathrm{~kb}$ from the TSS in mESCs ${ }^{16,40}$.

Motif analysis ${ }^{41}$ confirmed that the conserved PRDM14 motif was top ranking in both hESCs and hPGCLCs (Fig. 7c). Notably, TFAP2C motif was the second most enriched within hPGCLCspecific targets, suggesting cooperation between the two factors (Fig. 7c), consistent with the overlapping targets from RNA-seq (Supplementary Fig. 6F). We also found significant enrichment of BLIMP1 and SOX motifs in hPGCLCs, whereas unique hESC peaks were enriched in the OCT4-SOX2 motif (Fig. 7c). Altogether, this indicates that PRDM14 co-occupies genomic targets with core TFs specific to hPGCs or pluripotent cells, respectively.

Next, we correlated ChIP-seq peaks mapping within $100 \mathrm{~kb}$ of the TSS with the corresponding changes in gene expression from RNA-seq (Fig. 7d, Supplementary Fig. 6H and Supplementary Data 3). In hPGCLCs, 314 peaks were associated with $168(17 \%)$ upregulated genes and 93 peaks with $47(10 \%)$ downregulated genes (Supplementary Data 3). GO analysis confirmed that genes related to WNT signalling, heart and brain development are among the direct PRDM14 targets (Supplementary Fig. 7B). The 
a

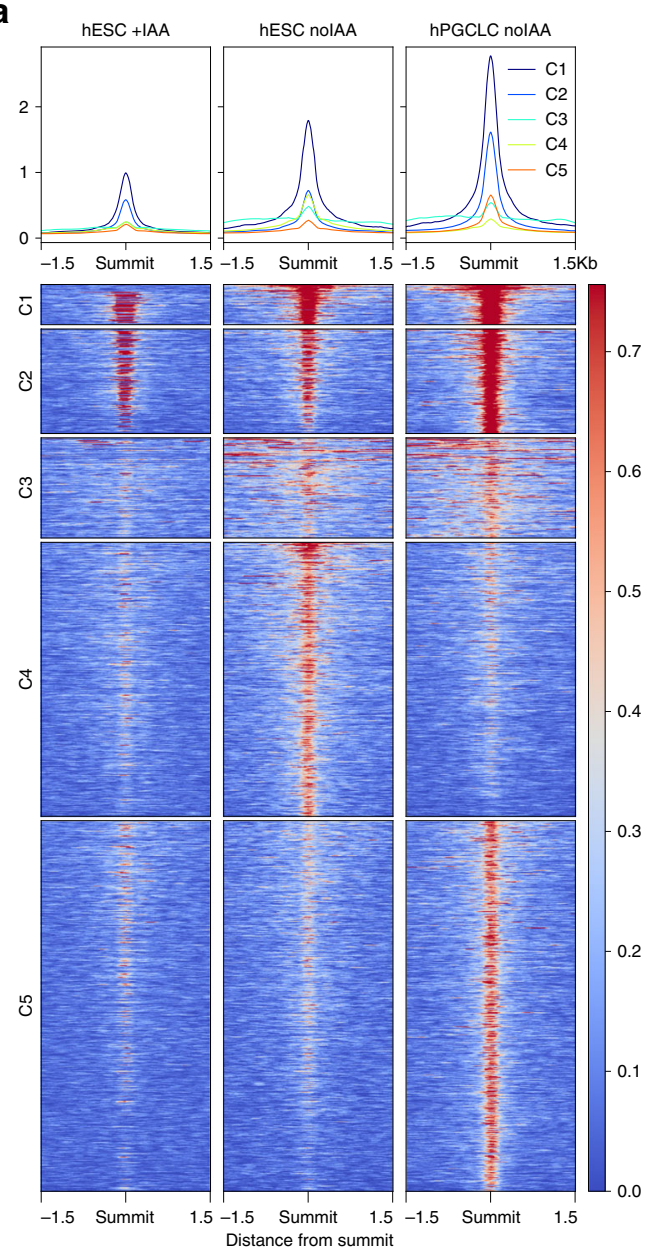

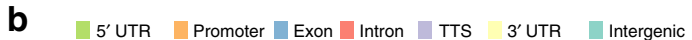

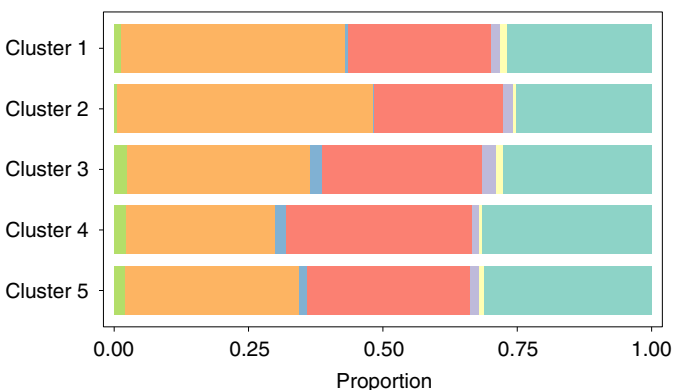

C

\begin{tabular}{|c|c|c|c|c|c|c|}
\hline \multicolumn{3}{|c|}{ hESC-specific peaks } & \multicolumn{4}{|c|}{ hPGCLC-specific peaks } \\
\hline Mouif & $p$-value & $\mathrm{TF}$ & Rank & Moiff & $\begin{array}{ll}p \text {-value } \\
\end{array}$ & $\mathrm{TF}$ \\
\hline-1 AGTACACGCCT & $1 E-133$ & PRDM14 & 18 & 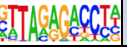 & $1 E-225$ & PRDM14 \\
\hline 3 TIGACATCAAp & $1 \mathrm{E}-39$ & OCT4-SOX2 & & 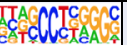 & 1E-179 & TFAP2C \\
\hline${ }_{4}$ CCITIGTCT & $1 E-30$ & sox11 & & ATACAATAG & $1 E-41$ & sox \\
\hline 5 TGGATCC & $1 E-19$ & TEAD3 & & AAGTGAAGTCT & 1E-19 & BLMP1 \\
\hline
\end{tabular}

d
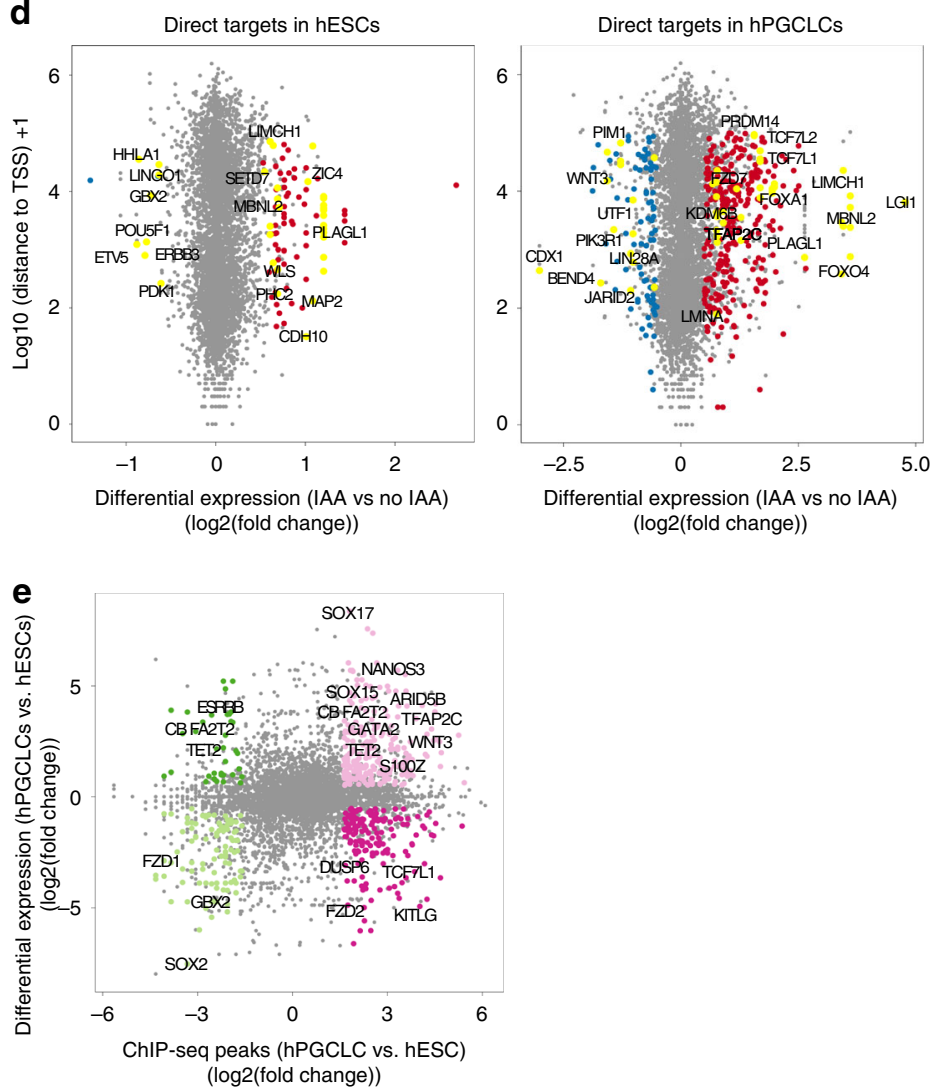

$\mathbf{f}_{\text {PAPA }}$

PRDM14 hESC

PRDM14 hESC nolAA

PRDM14 hPGCLC

PRDM14 hESC

(Chia)

Input hESC

Input hPGCLC

Input hESC

(Chia)

PRDM14 hESC

IIAA

PRDM14 hESC

nolAA

PRDM14 hPGCLC

PRDM14 hESC

(Chia)

Inputh ESC

Input hPGCLC

Input hESC

(Chia)
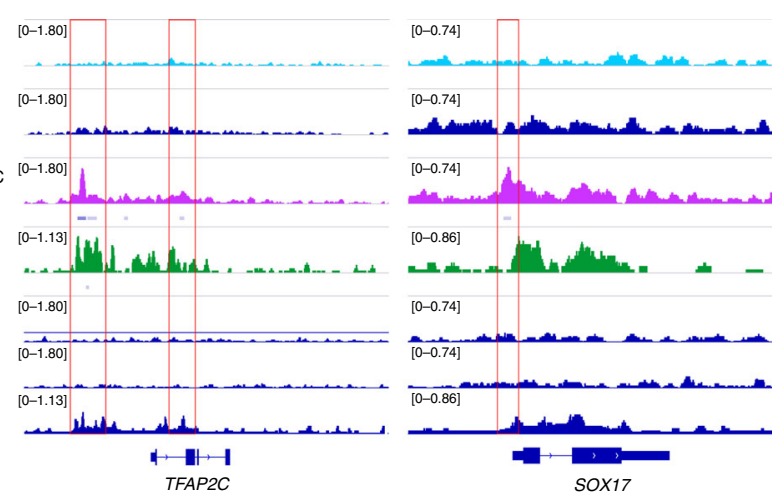

$[0-0.74]$
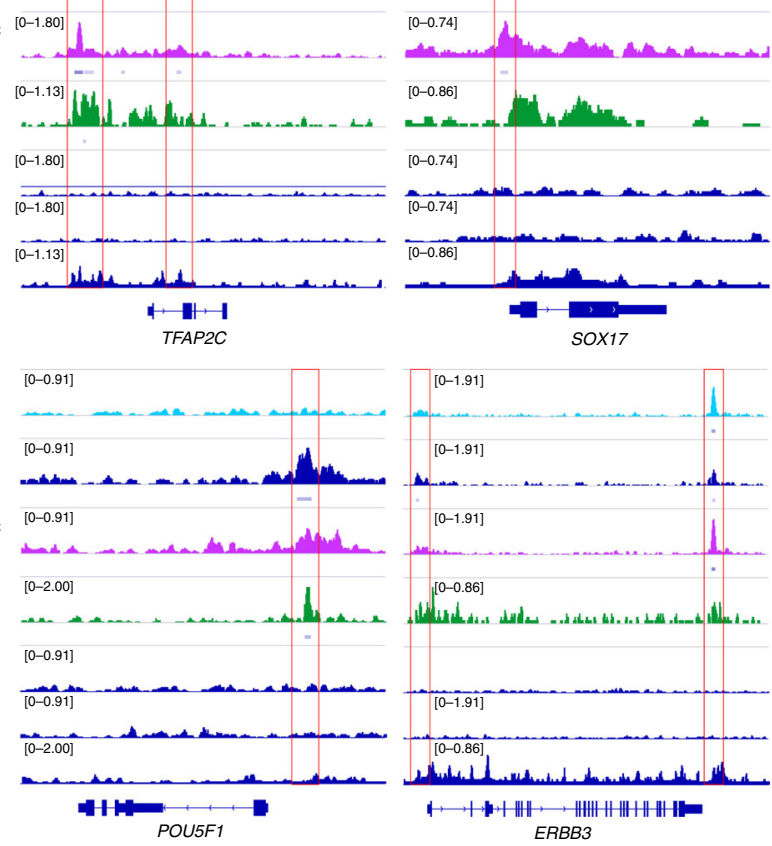

hESC-specific targets included SOX2, ERBB3 and GBX2, while hPGCLC-specific peaks were found near SOX17, TFAP2C and NANOS3 among others (Fig. 7e, f). Whereas PRDM14 binds to the regulatory elements of SOX17, PRDM14 depletion did not significantly affect SOX17 expression in hPGCLCs (Fig. 6e and
Supplementary Data 1) presumably because other factors sustain its expression.

Distinct molecular functions of PRDM14 in mouse and human. Next, we asked if the molecular roles of PRDM14 are 
Fig. 7 ChIP-seq analysis shows hESC- and hPGCLC-specific PRDM14 binding patterns. a Consensus PRDM14-bound peaks (6486) are separated into five clusters by $k$-means clustering. The ChIP signals (counts per million per 10-bp bin) of each peak are shown in the clustered heatmaps (bottom panel). The profile plots (top panel) show the average ChIP signals of each cluster. b Distribution of clustered PRDM14 peaks in the genome. Note that PRDM14 predominantly binds to promoters. c Top-ranking motifs identified by HOMER de novo motif analysis within hESC- and hPGCLC-specific PRDM14 peaks (FC > 3, $p$ value $<0.0001)$. d Scatter plots highlighting examples of direct PRDM14 targets in hESCs and hPGCLCs. Direct targets were defined as genes with at least one PRDM14 ChIP-seq peak ( $q$ value $<0.05$ ) within $100 \mathrm{~kb}$ of the TSS and showing significant changes in expression upon IAA treatment $(\log 2 \mathrm{FC}<(-0.5)$ or $>0.5$ and padj $<0.05)$. e PRDM14 binds to regulatory regions of key hPGC-related genes. The scatter plot shows differential PRDM14 peaks $(\mathrm{FC}>3, p$ value $<0.0001)$ correlated to genes differentially expressed between $\mathrm{hESCs}$ and $\mathrm{hPGCLCs}(\log 2 \mathrm{FC}<(-0.5)$ or $>0.5$ and padj $<0.05)$. f Examples of loci bound by PRDM14 in hPGCLCs and hESCs. Note that the peak downstream of ERBB3 is preserved in hESCs after IAA treatment. PRDM14 peaks in indicated samples were visualised using IGV software. PRDM14 ChIP-seq dataset from ref. ${ }^{13}$ was added for comparison.

conserved between mouse and human, by comparing the proteincoding transcriptomes of PRDM14-AID hESCs and hPGCLC, with the equivalent mouse $\operatorname{Prdm14^{-/}}{ }^{-}$cells $^{11}$ (Supplementary Fig. 8A, B and Supplementary Data 4). PGC-competent mouse epiblast-like cells showed very few DEGs since Prdm14 is repressed in these cells ${ }^{11}$. Only two genes (CDH4 and HS6ST2) were derepressed in both mESCs and hESCs, and three genes (DNMT3B, SPRY4 and FHL1) showed opposite changes, probably reflecting the broader differences between mESCs and hESCs ${ }^{42}$. Comparison of PRDM14-depleted hPGCLCs with D6 Prdm14 ${ }^{-/-}$ mPGCLC identified a limited subset of potentially conserved PRDM14 targets: 36 up- and 13 downregulated genes (Supplementary Fig. 8A), but none have hitherto been implicated in germ cell biology. Furthermore, the expression of 39 DEGs was anticorrelated in hPGCLC and mPGCLCs (Supplementary Fig. 8A).

In mPGCs, PRDM14 is key to epigenetic reprogramming, ensuring DNA demethylation through repression of Uhrf1 and loss of $\mathrm{H} 3 \mathrm{~K} 9 \mathrm{me} 2$ through Ehmt1 downregulation ${ }^{9}$. hPGCs display similar hallmarks of epigenetic reprogramming, associated with repression of $U H R F 1$ and EHMT2 (ref. ${ }^{26}$ ). While by RNA-seq we did not detect changes in UHRF1 or EHMT2 expression in PRDM14-deficient hPGCLCs, we verified UHRF1 and H3K9me2 levels by IF (Supplementary Fig. 8C, D), to exclude regulation at the protein level, as observed in other contexts ${ }^{43-45}$. This showed an increase in the proportion of $\mathrm{UHRF}^{+}$ proliferating D4 hPGCLCs upon PRDM14 depletion, potentially suggesting a conserved regulation (Supplementary Fig. 8C, G). The effect was, however, heterogeneous and there was no significant difference in mean fluorescence intensities of UHRF1 in proliferating hPGCLCs induced with or without IAA (Supplementary Fig. 8E). We did not detect significant retention of H3K9me2 in PRDM14-deficient hPGCLCs (Supplementary Fig. 8D, F, H). Overall this could suggest that PRDM14 is less critical for epigenetic reprogramming in hPGCs than it is in the mouse. However, D4 hPGCLCs model pre-migratory germ cells ${ }^{3}$, which might be too early to detect potential defects in epigenetic resetting caused by PRDM14 depletion.

PRDM14 alone is sufficient to induce mPGCLCs fate ${ }^{8}$; however, this was not the case for hPGCLC specification using either doxycycline-inducible or ProteoTuner systems (Supplementary Fig. 8I), consistent with the observation that upregulation of SOX17, BLIMP1 and TFAP2C precede PRDM14 expression (Fig. 2 and Supplementary Fig. 2). Altogether PRDM14 evidently plays an important role shortly after the initiation of hPGCLC fate, but its function is distinct from that in mPGCs.

\section{Discussion}

Using two acute protein depletion strategies, combined with rescue, transcriptomic and ChIP-seq experiments, we demonstrate that PRDM14 is required for hPGCLC specification and represses somatic differentiation while promoting germline fate (Supplementary Fig. 9). Strikingly, the molecular function of
PRDM14 in the human germline has diverged significantly compared with mPGCs. Indeed, the sets of targets regulated by PRDM14 in the two species are vastly different (Supplementary Fig. 8A, B) and, unlike in the mouse ${ }^{8}$, PRDM14 alone cannot induce hPGCLCs (Supplementary Fig. 8D). The regulatory network for hPGC specification is altogether distinct from that in mice, with the recently established critical role of SOX17, and the notable repression of SOX2 (refs. ${ }^{3,20)}$. Our study of PRDM14 provides further insights on the divergence of the molecular basis of germ cell fate determination in mouse and human.

PRDM14 is specifically upregulated in the nucleus of nascent hPGCLC, its expression follows that of SOX17, BLIMP1 and TFAP2C, but precedes the upregulation of KLF4 (Fig. 2 and Supplementary Fig. 2). PRDM14 depletion strongly reduces the efficiency of germ cell specification (Fig. 4) and results in an aberrant transcriptome of the PRDM14-deficient hPGCLCs (Fig. 6). Conversely, PRDM14 overexpression on D0 of induction rescues and even enhances hPGCLC specification (Fig. 5). Since hPGCLC induction is probably not a fully synchronous process, it is possible that PRDM14 overexpression in cells that start upregulating SOX17 and BLIMP1 might enhance their propensity to adopt hPGCLC fate through accelerating the expression of PRDM14 target genes, which perhaps helps consolidating germ cell identity.

The effects of PRDM14 depletion during hPGCLC specification, resemble those observed upon the loss of TFAP2C or BLIMP1 (Supplementary Fig. 6F). Furthermore, ChIP-seq analysis revealed high enrichment of TFAP2C and BLIMP1 motifs within hPGCLC-specific PRDM14 targets (Fig. 7c), which suggests combinatorial roles of the three regulators during the induction of hPGC-specific genes and the repression of somatic markers. However, unlike in mPGCs $^{11,46}$, many prominent germ cell genes, such as TFAP2C, PRDM1 and DND1, are not the targets of PRDM14 in hPGCLCs. Furthermore, while the PRDM14 motif is conserved, PRDM14 binds predominantly to gene promoters in hESCs and hPGCLCs ${ }^{13}$ (Fig. 7b), but to distal regulatory elements in mice ${ }^{16,40}$. The notable lack of overlap might be dictated by different protein partners or by the divergence of the protein itself, although there is significant functional conservation of the protein, as human PRDM14 rescues the lack of its mouse orthologue in mESCs ${ }^{15}$.

The context-dependent roles of PRDM14 are also exemplified by distinct set of target genes in hESCs, where it cooperates with OCT4 and SOX2 to sustain pluripotency, and limits neuronal differentiation (Fig. 6a, Supplementary Figs. 6E, 7C and 9). This and other studies highlighted the divergent roles of PRDM14 in mouse and human pluripotent cells ${ }^{13,15,16,40}$. Furthermore, there are significant human-mouse differences during early embryo development, at the time of PGC specification ${ }^{47,48}$; altogether these differences likely contribute to the species-specific modes of PGC specification. Notably, we found an even more pronounced transcriptional phenotype upon PRDM14 loss in hPGCLCs than in hESCs (Fig. 6). Note that hESCs were, however, in a 'steady state' of self-renewal, while hPGCLCs were undergoing cell fate 
transitions at the time of PRDM14 depletion. The persistence of PRDM14 at some loci even after 3 days of IAA treatment (Fig. 7a), is reminiscent of a significant (27\%) retention of CTCFAID peaks that were reported after 2 days of exposure to IAA ${ }^{34}$. Since most other inducible loss-of-function approaches display lower efficiency and slower kinetics, it is likely that they may result in even slower protein depletion from chromatin.

The distinct roles of PRDM14 in mouse and human PGCs, further illustrate the impact of the species-specific regulatory networks for PGC fate. For example, expression of SOX17 and SOX2 is mutually exclusive in germ cells of human and mouse, respectively. Whereas SOX17 is essential for hPGC fate ${ }^{3}$, SOX2 promotes mPGC specification and survival ${ }^{49}$. By contrast, repression of SOX2 is apparently prerequisite for the initiation of hPGCLC fate ${ }^{1}$, since ectopic expression of SOX2 abrogates hPGCLC specification ${ }^{50}$. In the absence of BMP signalling, which initiates hPGCLC specification, high expression of SOX2 and PRDM14 is maintained (Supplementary Fig. 2). It is possible that PRDM14 expression in the hPGCLC-competent cells is downstream of SOX2. The repression of SOX2 upon the initiation of hPGCLC in response to BMP signalling might therefore explain a transient loss of PRDM14. Indeed, in hESC where SOX2 binds to PRDM14 promoter ${ }^{51}$, loss of SOX2 leads to PRDM14 downregulation $^{52}$. The observed re-expression of PRDM14 in hPGCLC follows after the upregulation of SOX17 and BLIMP1 at the onset of hPGCLC specification (Fig. 2 and Supplementary Fig. 2), which suggests a molecular shift in the regulation of PRDM14 expression in germ cells.

In mPGCs, PRDM14 promotes DNA demethylation, in part by repressing $U h r f 1$, and initiates the reduction of $\mathrm{H} 3 \mathrm{~K} 9 \mathrm{me} 2$ through Ehmt1 downregulation ${ }^{9}$. How the TF network might control the epigenetic resetting in hPGCs is less clear, but some essential features of epigenetic reprogramming are evident in hPGCs, including the repression of UHRF1 and EHMT2 (ref. $\left.{ }^{26}\right)$. While there was a trend towards derepression of UHRF1 in a subset of PRDM14-deficient hPGCLCs (Supplementary Fig. 8C), we did not observe changes in $\mathrm{H} 3 \mathrm{~K} 9 \mathrm{me} 2$ levels. Unfortunately, currently available hPGCLCs only recapitulate pre-migratory hPGCs, and do not show significant epigenetic changes as observed in later-stage in vivo germ cells ${ }^{1}$. Therefore, it is difficult to comprehensively assess the influence of PRDM14 loss on epigenetic resetting in the human germline. Further studies are required to establish how precisely the epigenetic resetting is initiated in hPGCs and establish whether or not PRDM14 has an important role in this critical process.

Our study shows the importance of the use of rapid and comprehensive PRDM14 depletion for the phenotype unmasking. A previous study of PRDM14 in hPGCLCs used a partial knockdown at a later time-point (D2 $\mathrm{BLIMP} 1^{+} \mathrm{SOX} 17^{+}$precursors), where the homogeneity and speed of PRDM14 depletion was not monitored ${ }^{19}$. We demonstrate the utility of AID and JAZ degrons to deplete endogenous proteins to study human cell fate decisions. Simultaneous use of both degrons allows independent control of two proteins, and the construction of degron switches as shown here (Fig. 5b, d). The knock-in of AID/JAZ together with a fluorescent reporter facilitates protein expression analysis and allows the use of the anti-GFP (or other epitope tags) antibody for IF and ChIP. Furthermore, tissue-specific TIR1 expression can allow spatial control over protein stability, as shown in Caenorhabditis elegans and Drosophila melanogaster without detectable side effects ${ }^{53,54}$.

Neither mouse nor human adult tissues express PRDM14 except in some types of cancer ${ }^{14,55}$, indicating the importance of studying the role of PRDM14 in normal embryogenesis. Inducible degrons offer a more precise control over proteins when studying the roles of critical TFs undergoing dynamic changes during normal and malignant development.

\section{Methods}

Human embryonic stem cell culture. PGC-competent $4 \mathrm{i} \mathrm{hESCs}$ (H9 (ref. ${ }^{56}$ ), WIS2-NANOS3-T2A-tdTomato (N3) ${ }^{5}$ and cell lines derived from them) were cultured as in ref. ${ }^{3}$ on irradiated mouse embryonic fibroblasts (MEFs) (GlobalStem) in $4 \mathrm{i}$ medium (Table 1). Media were replaced every day. hESCs were passaged by single-cell dissociation using $0.25 \%$ Trypsin-EDTA (GIBCO). $10 \mu \mathrm{M}$ ROCK inhibitor (Y-27632, TOCRIS) was added for $24 \mathrm{~h}$ after passaging.

Conventional hESCs (used for hPGCLC differentiation via preME) were grown in Essential $8(\mathrm{E} 8)^{57}$ in plates pre-coated with $5 \mu \mathrm{g} / \mathrm{ml}$ vitronectin for at least $1 \mathrm{~h}$. Media were replaced every day. Cells were passaged in clumps using $0.5 \mathrm{mM}$ EDTA in PBS. All reagents for E8 hESC culture were from Thermo Fisher Scientific.

For transgene inductions in hESCs or during differentiation, $1 \mu \mathrm{g} / \mathrm{ml}$

doxycycline (dox, Sigma) and/or $0.5 \mu \mathrm{M}$ Shield-1 (Clontech) were added to media, where specified. For depletion of AID-fused proteins, auxin (indole-3-acetic acid sodium salt, IAA, Sigma) was used at $100 \mu \mathrm{M}$ (in $\mathrm{H}_{2} \mathrm{O}$ ) unless otherwise specified. For depletion of JAZ-fused proteins, coronatine (Cor, Sigma) was used at $50 \mu \mathrm{M}$ (in DMSO) in all experiments. DMSO was used as vehicle control for experiments with Cor.

HEK-293T cell manipulation. HEK-293T cells were cultured in DMEM with 10\% fetal bovine serum (FBS, Corning), $1 \mathrm{mM}$ sodium pyruvate, $2 \mathrm{mM}$ L-glutamine and PenStrep (all GIBCO) ${ }^{24}$. For JAZ degron testing, the cells were transfected with vectors for expression of GFP fused to $O s J \mathrm{AZ}^{33}$, OsJAZ ${ }^{33}$-NLS or $O s J \mathrm{AZ}^{43}$. Lentivirus production, HEK-293T transfection and selection were carried out as in ${ }^{24}$. The cells were then treated with either $50 \mu \mathrm{M}$ Cor (Sigma) in DMSO or $0.1 \%$ DMSO as control. GFP depletion was analysed using flow cytometry. FlowJo (TreeStar) was used to calculate the geometric mean of GFP fluorescence intensity.

hPGCLC and DE induction. To induce hPGCLCs ${ }^{3}$, $4 \mathrm{i} \mathrm{hESCs}$ were trypsinised, filtered and plated into ultra-low cell attachment U-bottom 96-well plates (Corning, 7007) at 4000 cells/well density in $100 \mu 1$ PGCLC medium ${ }^{5}$ (Table 2). For induction of large quantities of hPGCLCs (for ChIP-seq and qPCR experiments), six-well EZSPHERE microplates (ReproCELL) were used (500,000 cells/well in 3 $\mathrm{mL}$ PGCLC medium). The plates were centrifuged at $300 \mathrm{~g}$ for $3 \mathrm{~min}$ and placed into a $37^{\circ} \mathrm{C} 5 \% \mathrm{CO}_{2}$ incubator until embryoid body (EB) collection for downstream analysis. Reporter fluorescence intensities were monitored daily throughout differentiation using Olympus IX71 microscope.

For hPGCLC induction from E8 (conventional) $\mathrm{hESCs}^{5}$, preME competent state was induced by seeding 200,000 trypsinised single cells per well of a vitronectin-coated 12-well plate and culturing for $12 \mathrm{~h}$ in ME medium (Supplementary Table 1). PreME cells were then trypsinised, filtred and induced similar to $4 \mathrm{i}$ hESCs, as described above.

For DE induction ${ }^{5}, \mathrm{ME}$ was first obtained from $\mathrm{E} 8$ cells by 36 -h exposure to $\mathrm{ME}$ medium (Supplementary Table 1). ME was then washed with PBS followed by 48 -h culture in the DE medium (Supplementary Table 2).

Flow cytometry and fluorescence-activated cell sorting. Flow cytometry and FACS were performed as in ${ }^{3}$. The gating strategy for hPGCLC identification is shown in Supplementary Fig. 10. At least six EBs were washed in PBS and dissociated with $0.25 \%$ Trypsin-EDTA for $10 \mathrm{~min}$ at $37^{\circ} \mathrm{C}$. Cells were washed, resuspended in FACS buffer (3\% FBS in PBS) and incubated with anti-AP and antiCD38 antibodies specified in Supplementary Table 3 for $30-60 \mathrm{~min}$ at $4{ }^{\circ} \mathrm{C}$ in the dark. After washing, the cells were resuspended in FACS buffer with $0.1 \mu \mathrm{g} / \mathrm{ml}$ DAPI and filtered through a $50 \mu \mathrm{m}$ cell strainer. Flow cytometry was done using BD LSR Fortessa, while FACS was performed with Sony SH100 Cell Sorter. Data were analysed using FlowJo (Tree Star).

Isolation of gonadal hPGC by FACS. The collection and usage of human embryonic tissues were approved by the National Research Ethics Service (REC 96/ 085). Patients (who had already decided to undergo the termination of pregnancy operation) fully and freely consented to donate the foetal tissues for medical and academic research. Human genital ridges were dissected in PBS and separated from the mesonephros followed by dissociation with TrypLE Express (Life Technologies) at $37^{\circ} \mathrm{C}$ for $20-40 \mathrm{~min}$ (depending on the tissue size) with pipetting every $5 \mathrm{~min}$. Cells were washed, resuspended in FACS buffer (3\% FBS and $5 \mathrm{mM}$ EDTA in PBS) and incubated with anti-AP and anti-cKIT antibodies (specified in Supplementary Table 3) for $15 \mathrm{~min}$ at room temperature with $10 \mathrm{rpm}$ rotation in the dark. Cells were then washed in FACS medium and filtered through a $35 \mu \mathrm{m}$ cell strainer. FACS was performed with Sony SH100 Cell Sorter and data were analysed using FlowJo (Tree Star). Cell populations of interest were sorted onto Poly-L-Lysine Slides (Thermo Scientific) and fixed in 4\% PFA for IF analysis.

Immunofluorescence. For IF, hESCs were grown on ibiTreat eight-Well $\mu$-Slides (Ibidi) on MEFs (GlobalStem), washed with PBS and fixed with $4 \%$ paraformaldehyde (PFA, Thermo Fisher Scientific) in PBS at room temperature (RT) for 
Table 1 4i medium composition.

\begin{tabular}{lll} 
Component & $\begin{array}{l}\text { Final } \\
\text { concentration }\end{array}$ & Supplier \\
\hline $\begin{array}{l}\text { Knockout DMEM } \\
\text { Knockout serum } \\
\text { replacement (KSR) }\end{array}$ & - & GIBCO \\
L-glutamine & $20 \%$ & GIBCO \\
Nonessential amino acids & $2 \mathrm{mM}$ & GIBCO \\
2-mercaptoethanol & $0.1 \mathrm{mM}$ & GIBCO \\
Penicillin-streptomycin & $100 \mathrm{~m} / \mathrm{ml}$ & GIBCO \\
& $($ penicillin) & GIBCO \\
& $0.1 \mathrm{mg} / \mathrm{ml}$ & \\
Human LIF & $(\mathrm{streptomycin})$ & \\
& $20 \mathrm{ng} / \mathrm{ml}$ & Stem Cell \\
bFGF & & Institute (SCI) \\
TGF- $\beta 1$ & $8 \mathrm{ng} / \mathrm{ml}$ & SCI \\
CHIR99021 (CH) & $1 \mathrm{ng} / \mathrm{ml}$ & Peprotech \\
PD0325901 (PD) & $3 \mu \mathrm{M}$ & Miltenyi Biotec \\
SB203580 (SB) & $1 \mu \mathrm{M}$ & Miltenyi Biotec \\
SP600125 (SP) & $5 \mu \mathrm{M}$ & TOCRIS bioscience \\
\hline
\end{tabular}

Table 2 hPGCLC medium composition.

\begin{tabular}{lll} 
Component & Final concentration & Supplier \\
\hline Advanced RPMI 1640 & - & GIBCO \\
B27 supplement & $1 \%$ & GIBCO \\
L-glutamine & $2 \mathrm{mM}$ & GIBCO \\
Nonessential amino acids & $0.1 \mathrm{mM}$ & $\mathrm{GIBCO}$ \\
Penicillin-streptomycin & $100 \mathrm{U} / \mathrm{ml}$ (Penicillin) & $\mathrm{GIBCO}$ \\
& $0.1 \mathrm{mg} / \mathrm{ml}$ & \\
BMP2 & $($ Streptomycin) & \\
Human LIF & $500 \mathrm{ng} / \mathrm{ml}$ & $\mathrm{SCl}$ \\
SCF & $1 \mu \mathrm{g} / \mathrm{ml}$ & $\mathrm{SCl}$ \\
EGF & $100 \mathrm{ng} / \mathrm{ml}$ & R\&D systems \\
ROCK inhibitor (Y-27632) & $50 \mathrm{ng} / \mathrm{ml}$ & R\&D systems \\
& $10 \mu \mathrm{M}$ & TOCRIS \\
Poly-vinyl alcohol (PVA, 10\%) & $0.25 \%$ & bioscience \\
\end{tabular}

$10 \mathrm{~min}$. This was followed by three washes in PBS and a 10-min permeabilisation in $0.25 \%$ Triton X-100 (Sigma) in PBS. Next, the samples were incubated with the blocking buffer $(0.1 \%$ Triton X-100, 5\% normal donkey serum (Stratech) and $1 \%$ bovine serum albumin (BSA, Sigma)) for $30 \mathrm{~min}$ at RT. The samples were then incubated with primary antibodies (Supplementary Table 3) diluted in the blocking buffer overnight at $4{ }^{\circ} \mathrm{C}$. The following day they were washed 3 times with the wash buffer ( $0.1 \%$ Triton X-100 in PBS) and incubated with Alexa fluorophore (AF- 488, 568 and/or 647)-conjugated secondary antibodies (Invitrogen, used in 1:500 dilutions in the blocking buffer) specific for the host species of the primary antibodies for $1 \mathrm{~h}$ at RT in the dark. After three washes in the wash buffer, the samples were incubated in PBS with $1 \mu \mathrm{g} / \mathrm{ml}$ DAPI for $10 \mathrm{~min}$ at RT. The samples were stored at $4{ }^{\circ} \mathrm{C}$ in the dark (up to 2 weeks) before imaging on Leica SP5 inverted confocal microscope. The images were analysed using Fiji software.

hPGCLC-containing EBs were fixed on D4 (unless otherwise specified) using $4 \%$ PFA in PBS at $4{ }^{\circ} \mathrm{C}$ for $1-2 \mathrm{~h}$. After two washes in PBS, the EBs were transferred to $10 \%$ sucrose solution in PBS and stored at $4{ }^{\circ} \mathrm{C}$ for 1 day. This was followed by a 1-day incubation in $20 \%$ sucrose in PBS at $4^{\circ} \mathrm{C}$. Finally, the EBs were embedded in OCT compound (CellPath), snap frozen on dry ice and stored at $-80^{\circ} \mathrm{C}$ until cryosectioning. Cryosections of $8-\mu \mathrm{m}$ thickness were made on Superfrost Plus Micro slides (VWR) using a Leica Microsystems cryostat. Slides were stored at $-80^{\circ} \mathrm{C}$ or processed immediately. For IF, slides were air-dried at RT for $1 \mathrm{~h}$, washed in PBS three times 5 min each and permeabilised in $0.1 \%$ Triton X-100 in PBS for $30 \mathrm{~min}$ at RT. The cryosections on each slide were then circumscribed using ImmEdge Hydrophobic Barrier Pen (Vector Labs) and blocking solution (as above) was added for $30 \mathrm{~min}$ at RT. Samples were then incubated with primary and secondary antibodies (Supplementary Table 3 ) as specified for hESC IF above, but $1 \mu \mathrm{g} / \mathrm{ml}$ DAPI was added to the secondary antibody mixtures. Finally, the slides were washed in the wash buffer twice and in PBS once and mounted with Prolong
Gold Antifade Reagent (Molecular Probes). The images were acquired using Leica SP5 confocal microscope and analysed using Fiji software ${ }^{58}$.

A custom script for Fiji written by Dr Richard Butler (Gurdon Institute, University of Cambridge, UK) was used to segment nuclei with an area of $30-300 \mu \mathrm{m}^{2}$ in the DAPI channel and measure fluorescence intensity in other channels. The script is available at https://github.com/gurdon-institute/SOX17_PRDM14_Measurement. The data was then manually processed in Microsoft Excel to filter pluripotent cells (NANOG or OCT4 fluorescence intensity $>50$ ) where applicable and to calculate mean fluorescence intensities. Statistical analyses were performed using GraphPad Prism 7.

Plasmid constructions and cell line establishment. For gene targeting, CRISPRCas9 approach was used ${ }^{25}$. Annealed oligos (Supplementary Data 5) encoding corresponding gRNAs were ligated into pX330 vector ${ }^{25}$ digested with BbsI. gRNA sequences were chosen using the MIT CRISPR tool (http://www.genomeengineering.org/crispr/) to be located close to the stop codon (to enable C-terminal fusions in PRDM14-T2A-Venus, PRDM14-AID-Venus, PRDM14-JAZ-Venus and SOX17-AID-Venus). The chosen protospacer sequences were as follows: GTGAAGACTACTAGCCCTGC for PRDM14 and GACGTGTGACAGGTCCC TGA for SOX17 (Naoko Irie, personal communication).

All other plasmids, including donor vectors for homology-directed repair were generated using In-Fusion cloning (Clontech) according to manufacturer's recommendations, but scaling down the reaction to a total volume of $5 \mu$ l. Primers used for cloning are specified in Supplementary Data 5.

For knock-ins either electroporation or lipofection was used. For electroporation, $\sim 250,000$ trypsinised hESCs were resuspended in $600 \mu \mathrm{lBS}+$ $\mathrm{Ca}^{2+}+\mathrm{Mg}^{2+}$ containing $50 \mu \mathrm{g}$ gRNA plasmid and $50 \mu \mathrm{g}$ homologous repair donor plasmid and electroporated in a $0.4-\mathrm{cm}$ cuvette using Gene Pulser Xcell System (Bio-Rad) with a single $20 \mathrm{~ms}$ square-wave pulse $(250 \mathrm{~V})$. Lipofections were performed using $2 \mu \mathrm{g}$ gRNA plasmid and $2 \mu \mathrm{g}$ donor construct in OptiMEM medium (GIBCO) and Lipofectamine Stem reagent (Invitrogen) according to manufacturer's recommendations. The volume of lipofectamine in microlitres was equal to the total amount of DNA in micrograms. For lipofection of PiggyBac transgenes a total of 1-5 $\mu \mathrm{g}$ DNA/100,000 cells was used, with the amount of PiggyBac transposase (PBase)-encoding plasmid equal to that of PiggyBacdelivered transgenes combined (in $\mu \mathrm{g}$ ). Transfected cells were seeded onto drugresistant DR4 MEFs (SCI) in 4i medium (ROCKi added for the first $24 \mathrm{~h}$; selection was initiated $48 \mathrm{~h}$ after transfection).

After selection, individual clones were picked, expanded and genotyped using PCR. For this, genomic DNA was first extracted from cell pellets in the lysis buffer (10 mM Tris pH 8.0, $100 \mathrm{mM} \mathrm{NaCl}, 10 \mathrm{mM}$ EDTA and 0.5\% SDS; Proteinase K (PK) was added immediately prior to lysis at a final concentration of $0.2 \mathrm{mg} / \mathrm{ml}$ ) at $56^{\circ} \mathrm{C}$ for $4 \mathrm{~h}-$ overnight, followed by PK inactivation at $98^{\circ} \mathrm{C}$ for $10 \mathrm{~min}$. The supernatant was used directly in genotyping PCR performed with LongAmp (NEB) or PrimeStar GXL (Clontech) polymerase according to the manufacturers' protocols. Primers used for genotyping are specified in Supplementary Data 5. In the case of transgenes expression, homogeneity and leakiness of induction was checked by IF.

For knock-ins (PRDM14-T2A-Venus, PRDM14-AID-Venus, PRDM14-JAZVenus and SOX17-AID-Venus) correct targeting in homozygous (by genotyping) clones was confirmed by Sanger sequencing. This was followed by the removal of the Rox-flanked puromycin resistance cassette by transient transfection with $1 \mu \mathrm{g}$ of Dre-recombinase-encoding plasmid ${ }^{59}$. Two days of hygromycin B selection (50 $\mu \mathrm{g} / \mathrm{ml})$ were followed by 2 days of negative selection using FIAU $(200 \mathrm{nM})^{28}$. The obtained clones were again genotyped by PCR and sequenced to confirm antibiotic cassette excision. Venus intensity and homogeneity were then verified using Flow cytometry and IF.

For generation of cell lines that deplete PRDM14 upon IAA supplementation, AID-Venus was added at the C-terminus of PRDM14 using CRISPR (see above). This was followed by the addition of TIR1 transgene (pPB-CAG-OsTir1-mycIRES-HygR or pPB-CAG-OsTir1-V5-T2A-Puro) by lipofection, along with pPBase. TIR1 cDNA and the 44-amino-acid AID sequence ${ }^{34}$ were kindly provided by Dr Elphège Nora.

For generation of cell lines that deplete PRDM14 upon Cor supplementation, JAZ-Venus was added at the C-terminus of PRDM14 using CRISPR (see above). This was followed by the addition of COI1B transgene (pPB-CAG-HA-

FboxOsTir1-OsCoilb-IRES-HygR) by lipofection, along with pPBase.

For PRDM14-DD rescue, PRDM14-AID-Venus + TIR1 hESCs were colipofected with $0.05 \mu \mathrm{g}$ pPB-CAG-myc-PRDM14-DD-IRES-HygR (low DNA amount was used to limit transgene copy number), $0.5 \mu \mathrm{g}$ pPB-CAG-OsTir1-V5T2A-Puro (to avoid TIR1 excision by PBase) and $0.55 \mu \mathrm{g}$ PBase.

Reverse-transcription quantitative PCR (qPCR). Total RNA was extracted using the RNeasy Mini Kit (QIAGEN) from unsorted cells or using Arcturus PicoPure (Thermo Fisher Scientific) from at least 1000 sorted cells. cDNA was synthesised using the QuantiTect Reverse-Transcription Kit (QIAGEN). qPCR was performed on a QuantStudio 12K Flex Real-Time PCR machine (Applied Biosystems) using SYBR Green JumpStart Taq ReadyMix (Sigma) and specific primers (Supplementary Data 5). The $\Delta \Delta \mathrm{Ct}$ method was used for quantification of gene expression. 
Statistical analyses were performed using Microsoft Excel and/or GraphPad Prism 7.

RNA-sequencing. RNA-seq was performed on PRDM14-AID-Venus competent $4 \mathrm{i}$ hESCs and hPGCLCs induced therefrom. Two biological replicates were used for each condition. For IAA-sensitive cells, two clones (cl11 and cl21) from the same hESC passage or the same hPGCLC induction were used as replicates. For the parental (no TIR1) control, the same cell line was used at different passages or inductions to yield two independent replicates. In total, 10,000 $\mathrm{AP}^{+} 4 \mathrm{i} \mathrm{hESCs}$ or 10,000 NANOS3-tdTomato ${ }^{+} \mathrm{AP}^{+}$hPGCLCs (with the exception of hPGCLC cl21 replicate, where 3000 cells were used) were sorted directly into $100 \mu$ of extraction buffer from the PicoPure RNA Isolation Kit (Applied Biosystems) for subsequent total RNA extraction according to manufacturer's protocol. RNA was stored at $-80^{\circ} \mathrm{C}$ and its quality and quantity were checked by the Agilent RNA 6000 Pico Kit with Bioanalyzer (Agilent Technologies) and Qubit (Thermo Fisher Scientific). RNA-seq library was prepared from $10 \mathrm{ng}$ input RNA using the end-to-end Trio RNA-seq library prep kit (Nugen) following the manufacturer's protocol but omitting the AnyDeplete step. In short, the protocol contains the following steps: DNAse treatment to remove DNA from RNA; first strand and second strand cDNA synthesis to produce the reverse complement of the input RNAs; cDNA purification using Agencourt AMPure XP beads (Beckman Coulter); single-primer isothermal amplification to stoichiometrically amplify cDNAs; enzymatic fragmentation and end repair; sequencing adaptor (index) ligation; product purification using AMPure beads; library amplification (four cycles were used); library purification using AMPure beads. Libraries were then quantified by qPCR using the NEBNExt Library Quant Kit (NEB) for Illumina on QuantStudio 6 Flex RealTime PCR System (Applied Biosystems). Fragment size distribution and the absence of adaptor dimers was checked using Agilent TapeStation 2200 and High Sensitivity D1000 ScreenTape. Finally, RNA-seq libraries were subjected to singleend $50 \mathrm{bp}$ sequencing on HiSeq 4000 sequencing system (Illumina). Twenty-four indexed libraries (including samples from this work and eight others) were multiplexed together and sequenced in two lanes of a flowcell, resulting in $>30$ million reads per sample.

RNA-seq analysis. The library sequence quality in demultiplexed fastq files was checked by FastQC (v0.11.5) ${ }^{60}$ and the low-quality reads and adaptor sequences were removed by Trim Galore (v0.4.1) ${ }^{61}$ using the default parameters. The preprocessed RNA-seq reads were mapped to the human reference genome (UCSC GRCh38/hg38) using STAR (2.6.0a) ${ }^{62}$ (-outFilterMismatchNoverLmax 0.05 outMultimapperOrder Random -winAnchorMultimapNmax 100 -outFilterMultimapNmax 100) guided by the ENSEMBL (Release 87) gene models. Read counts per gene were extracted using TEtranscripts ${ }^{63}$ and normalised by DEseq2 in $\mathrm{R}^{64}$. Differential expression analysis was also performed using DEseq2. The resulting gene expression table (Supplementary Data 1) was used for downstream analyses in Microsoft Excel and R. Pearson's correlation analysis was performed using the $\mathrm{R}$ cor command. Unsupervised hierarchical clustering was performed using the $\mathrm{R}$ hclust function with (1-Pearson's correlation coefficient) as distance measures. The R prcomp function was used for PCA. GSEA was performed using the GSEA software by the Broad Institute ${ }^{65}$. GO analysis was performed using DAVID ${ }^{66}$

For comparative differential expression analysis of PRDM14 depletion in hPGCLCs and TFAP2C and PRDM1 KOs from ${ }^{20}$ the samples were identically preprocessed and mapped to the human reference genome (UCSC GRCh38/hg38), sequencing reads were re-normalised together using DEseq2 to generate a new integrated gene expression table (Supplementary Data 2). A similar procedure was performed for comparisons with mouse Prdm14 KO from ${ }^{11}$, where processed reads were mapped to the mouse reference genome (UCSC GRCm $38 / \mathrm{mm} 10$ ), and highconfidence human-mouse one-to-one orthology assignments for protein-coding genes were obtained from ENSEMBL (Release 87) (Supplementary Data 4). Venn diagrams were plotted using VennPainter ${ }^{67}$ and $p$ values for two overlapping datasets were calculated using a generalised hypergeometric test for multiple samples $^{68}$. For the co-expression analysis of DEGs, the gene-based Pearson's correlation coefficients were calculated for PRDM14-AID-Venus competent hESC and hPGCLC samples using R. All pairwise correlations $r<0.8$ between genes were removed. The matrix was imported as an adjacency matrix into the $\mathrm{R}$ igraph package ${ }^{69}$, and the Louvain method for community detection was performed on the resulting graph. For comparison with microarray data ${ }^{13}$, the published data was normalised, $\log 2$-transformed and the differential expression was evaluated using the R limma package ${ }^{70}$.

Chromatin immunoprecipitation (ChIP) and ChIP-seq. ChIP was performed using the SimpleChIP Enzymatic Chromatin IP Kit (with Magnetic Beads) from Cell Signalling Technology (CST) following manufacturer's recommendations with modifications. Recipes for all buffers are provided in Table 3. The following samples were included in the analysis (one replicate per condition): 4i hESCs (cl11 no IAA), $4 \mathrm{i}+$ IAA hESCs (cl11 + IAA) and hPGCLCs (cl11 and cl21 pooled; no IAA). For each ChIP 2.5 million unsorted 4i hESCs or 4 million unsorted hPGCLCs (D4 EBs containing 60-70\% PGCLCs as assessed by flow cytometry) were used. The cells were washed in PBS, filtered through $50 \mu \mathrm{m}$ strainer and fixed
Table 3 Buffer recipes for ChIP.

Buffer A

Buffer A stock, $\mu \mathrm{l}$

$\mathrm{H}_{2} \mathrm{O}, \mu \mathrm{l}$

DTT, $\mu \mathrm{l}$

PIC, $\mu \mathrm{I}$

Buffer B

Buffer B stock, $\mu \mathrm{l}$

$\mathrm{H}_{2} \mathrm{O}, \mu \mathrm{l}$

$\begin{array}{ll}\text { DTT, } \mu \mathrm{l} & 0.55\end{array}$

ChIP buffer $1 \quad 1 \mathrm{x}$

ChIP buffer stock, $\mu \mathrm{l}$

$\mathrm{H}_{2} \mathrm{O}, \mu \mathrm{l}$

PIC, $\mu$ I

0.5

ChIP buffer 2

$1 \mathrm{x}$

ChIP buffer stock, $\mu \mathrm{l}$

$\mathrm{H}_{2} \mathrm{O}, \mu \mathrm{l}$

360

PIC, $\mu$ l

tRNA (Sigma) $[10 \mathrm{mg} / \mathrm{ml}], \mu \mathrm{l}$

2

Elution

Elute buffer, $\mu \mathrm{l}$

$\mathrm{H}_{2} \mathrm{O}, \mu \mathrm{l}$

Blocking buffer

ChIP buffer (CST), $\mu$ l

$\mathrm{H}_{2} \mathrm{O}, \mu \mathrm{l}$

PIC (Roche), $\mu$ I

BSA (Sigma) (10\%), $\mu$

tRNA (Sigma) $[10 \mathrm{mg} / \mathrm{ml}], \mu \mathrm{l}$

Low salt

ChIP buffer (CST), $\mu$ l

$\mathrm{H}_{2} \mathrm{O}, \mu \mathrm{l}$

High salt

ChIP buffer (CST), $\mu$ l

$\mathrm{H}_{2} \mathrm{O}, \mu \mathrm{l}$

$\mathrm{NaCl}, \mu \mathrm{l}$

LiCl Wash buffer

$20 \mathrm{mM}$ Tris- $\mathrm{HCl} \mathrm{pH} \mathrm{8.0,} \mathrm{ml}$

2 mM EDTA, ml

$250 \mathrm{mM} \mathrm{LiCl}, \mathrm{g}$

$1 \%$ NP-40, ml

$1 \%$ Deoxycholate, $g$

$\mathrm{H} 2 \mathrm{O}, \mathrm{ml}$

2

$1 \mathrm{x}$

25

25

$1 \mathrm{x}$

150

1110

75

150

15

$1 \mathrm{x}$

300

2700

$1 \mathrm{x}$

100

900

70

For $250 \mathrm{~mL}$

$5 \mathrm{ml}$ of $1 \mathrm{M}$

$1 \mathrm{ml}$ of $0.5 \mathrm{M}$

$2.65 \mathrm{~g}$

$2.5 \mathrm{ml}$

$2.5 \mathrm{~g}$

$241.5 \mathrm{ml}$

in $1 \%$ formaldehyde at RT for 10 min. Formaldehyde was quenched by adding 450 $\mu \mathrm{l}$ of $10 \times$ glycine at RT for $10 \mathrm{~min}$, followed by centrifugation at $500 \mathrm{~g}, 4^{\circ} \mathrm{C}$ for 5 $\mathrm{min}$. The cells were then washed twice in ice-cold PBS with protease inhibitor cocktail (PIC) and the pellets were snap frozen on dry ice and stored at $-80^{\circ} \mathrm{C}$. To prepare nuclei, cells were thawed on ice, washed in ice-cold buffers A and B, resuspended in $100 \mu \mathrm{l}$ buffer $\mathrm{B}$ and transferred to a thin-walled Diagenode $1.5 \mathrm{ml}$ tubes. Next, $0.35 \mu \mathrm{l}$ micrococcal nuclease (MNase) was added and the suspension was incubated for $23 \mathrm{~min}$ at $37^{\circ} \mathrm{C}$, shaking at $800 \mathrm{rpm}$ to enzymatically digest the chromatin. The addition of $10 \mu \mathrm{l} 0.5 \mathrm{M}$ EDTA for $2 \mathrm{~min}$ on ice was used to stop the digestion. Next, the nuclei were pelleted at $15,500 \mathrm{~g}, 4^{\circ} \mathrm{C}$ for $1 \mathrm{~min}$, resuspended in $100 \mu \mathrm{l}$ of ChIP buffer 1 and kept on ice for $10 \mathrm{~min}$, followed by sonication $(3 \times 30 \mathrm{~s}$ on/off, high output) using Bioruptor sonicator (Diagenode). The lysate was cleared by centrifugation at $9200 \mathrm{~g}, 4^{\circ} \mathrm{C}$ for $10 \mathrm{~min}$ and transferred to a fresh DNA LoBind $1.5 \mathrm{ml}$ tube (Eppendorf). For ChIP, $400 \mu \mathrm{l} \mathrm{ChIP} \mathrm{buffer} \mathrm{two} \mathrm{was} \mathrm{added} \mathrm{for} \mathrm{a} \mathrm{total} \mathrm{of}$ $500 \mu \mathrm{l} /$ sample; $10 \mu \mathrm{l}$ were removed and stored at $-80^{\circ} \mathrm{C}$ to serve as $2 \%$ input. Antibodies ( $3 \mu$ l PRDM14 ABD121 or $0.5 \mu$ GFP ab290, see Supplementary Table 3) were then added to each immunoprecipitation (IP) reaction and IPs were incubated overnight rotating at $10 \mathrm{rpm}, 4^{\circ} \mathrm{C}$.

Equal volumes of Protein A and Protein G-conjugated magnetic beads (Invitrogen) were combined (for a total of $20 \mu \mathrm{l}$ per IP) in a DNA LoBind $1.5 \mathrm{ml}$ tube, washed in $200 \mu \mathrm{l}$ blocking buffer, and incubated with $500 \mu \mathrm{l}$ blocking buffer for $1 \mathrm{~h}$ rotating at $10 \mathrm{rpm}, 4^{\circ} \mathrm{C}$. The tubes were placed on a magnetic rack (Diagenode) to remove the supernatant and another 1-h incubation was performed. Then the supernatant was removed, the beads were resuspended in 20 $\mu \mathrm{l}$ blocking buffer and stored at $4{ }^{\circ} \mathrm{C}$ for up to $24 \mathrm{~h}$ until the use in ChIP.

After overnight IP, $20 \mu \mathrm{l}$ of pre-blocked Protein A/G beads were added to each sample, followed by rotation at $10 \mathrm{rpm}, 4^{\circ} \mathrm{C}$ for $2 \mathrm{~h}$. The tubes were placed on a magnetic rack and the supernatant was removed. The beads were then washed three times with $1 \mathrm{ml}$ low-salt buffer (each wash involved a 5 -min rotation at $10 \mathrm{rpm}, 4^{\circ} \mathrm{C}$ ), 
once with high-salt buffer and once with $\mathrm{LiCl}$ buffer. Finally, the beads were resuspended in $50 \mu \mathrm{l}$ elution buffer and transferred to $0.2 \mathrm{ml}$ PCR stripes. The inputs were thawed on ice and supplemented with $50 \mu \mathrm{l}$ elution buffer. All samples were incubated at $65^{\circ} \mathrm{C}, 110 \mathrm{~g}$ for $30 \mathrm{~min}$ to elute the protein-DNA complexes from the beads. The stripe was then placed onto a magnetic rack and the eluate was transferred into a fresh $0.2 \mathrm{ml}$ PCR stripe to which $2 \mu \mathrm{l} 5 \mathrm{M} \mathrm{NaCl}$ and $2 \mu \mathrm{l} \mathrm{PK}(10 \mathrm{mg} / \mathrm{ml})$ were added. The samples were then incubated at $65^{\circ} \mathrm{C}, 110 \mathrm{~g}$ for $2 \mathrm{~h}$ for decrosslinking.

Finally, for the use in ChIP-qPCR, DNA was purified using MinElute columns (QIAGEN) following the manufacturer's protocol. Alternatively, for ChIP-seq library preparation, DNA was purified using Agencourt AMPure XP beads (Beckman Coulter) as follows. 1.8 $\times$ volume of AMPure beads were mixed with each sample, incubated for $10 \mathrm{~min}$ to bind DNA to the beads and washed $2 \times 30 \mathrm{~s}$ with $200 \mu \mathrm{l} 80 \%$ ethanol using a magnetic rack. The beads were air-dried for 5-10 min to reach complete ethanol evaporation. Thirty three microlitres of elution buffer (10 $\mathrm{mM}$ Tris- $\mathrm{HCl}, \mathrm{pH} 8.0-8.5)$ was added and incubated with the beads for 2 min to elute the ChIP DNA. The PCR stripe was placed on the magnetic rack and $30 \mu \mathrm{l}$ of eluate was transferred into fresh tubes.

For ChIP-seq libraries were prepared using the KAPA HyperPrep Kit following the manufacturer's instructions. Briefly, the protocol contains the following steps: end-repair and A-tailing; sequencing adaptor (index) ligation; product purification using AMPure beads; library amplification using the KAPA real-time Library Amplification Kit (11 cycles were used for ChIP libraries and 7 for input libraries to achieve similar concentration range); product purification using AMPure beads. Libraries were then quantified by qPCR using the NEBNExt Library Quant Kit for Illumina (NEB) on QuantStudio 6 Flex Real-Time PCR System (Applied Biosystems). Fragment size distribution and the absence of adaptor dimers was checked using Agilent TapeStation 2200 and High Sensitivity D1000 ScreenTape. Finally, ChIP-seq libraries were subjected to single-end 50 bp sequencing on HiSeq 4000 sequencing system (Illumina). Eight indexed libraries were multiplexed in one lane of a flowcell, resulting in $>20$ million reads per sample.

ChIP-seq analysis. The library sequence quality in demultiplexed fastq files was checked by FastQC (v0.11.5) ${ }^{60}$ and the low-quality reads and adaptor sequences were removed by Trim Galore (v0.4.1) ${ }^{61}$ using the default parameters. The trimmed ChIPseq reads were aligned to the human reference genome (UCSC hg38) by the Burrows-Wheeler Aligner (0.7.15-r1142-dirty) ${ }^{71}$. Samtools (version: 1.3 .1$)^{72}$ was used to remove unmapped and low-mapping quality reads (options: view -F 4 -q 20). Subsequently, duplicated reads and reads mapped to unlocalised contig (random), unplaced contigs (ChrUn) and blacklisted regions (obtained from http://mitra. stanford.edu/kundaje/akundaje/release/blacklists/hg38-human/) were removed using Samtools rmdup. This was followed by preliminary peak calling using macs 2 callpeak ${ }^{73}$ against the corresponding inputs (options: -g $3 \mathrm{e} 9$-keep-dup all). Percentage of reads in peaks was calculated using featureCounts ${ }^{74}$ and visualised with multiQC ${ }^{75}$. Before peak calling, to compare the number of peaks between the three high-quality samples, the library size for all libraries was downsampled to 25 million reads. Peaks were then called again using macs 2 callpeak against the corresponding inputs (options: -g 3e9 -keep-dup all -nomodel -extsize 157). The resultant peak files of the three samples were merged to generate a consensus set of 6486 peaks using bedtools merge (options: $-\mathrm{d} \mathrm{100})^{76}$. The consensus peaks were processed and clustered by DeepTools computeMatrix and plotHeatmap ${ }^{77}$. Peak annotation, motif enrichment and differential peak analyses were performed by HOMER ${ }^{41}$. DeepTools bamCoverage (options: -extendReads 157 -binSize 10 -normalizeUsing CPM -ignoreForNormalization chrX chrY) was used to generate bigwig file for visualisation in Integrative Genomics Viewer ${ }^{78}$. The integrated ChIP-seq and RNA-seq table (Supplementary Data 3) was generated using $\mathrm{R}$ to correlate changes in gene expression to PRDM14 binding. Venn diagrams were plotted using the R/Bioconductor ChIPseeker package $^{79}$.

Reporting summary. Further information on research design is available in the Nature Research Reporting Summary linked to this article.

\section{Data availability}

All relevant data, including full plasmid sequences, are available from the authors on request. The source data underlying experimental results for Fig. $1 \mathrm{~d}$ is provided as a Source Data file. High-throughput sequencing (RNA-seq and ChIP-seq) data have been deposited at the NCBI Gene Expression Omnibus (GEO) database and can be found at the following address: https://www.ncbi.nlm.nih.gov/geo/query/acc.cgi?acc=GSE138675.

Received: 1 February 2019; Accepted: 31 December 2019;

Published online: 09 March 2020

\section{References}

1. Tang, W. W., Kobayashi, T., Irie, N., Dietmann, S. \& Surani, M. A. Specification and epigenetic programming of the human germ line. Nat. Rev. Genet. 17, 585-600 (2016).
2. Kobayashi, T. \& Surani, M. A. On the origin of the human germline. Development 145, dev150433, https://doi.org/10.1242/dev.150433 (2018).

3. Irie, N. et al. SOX17 is a critical specifier of human primordial germ cell fate. Cell 160, 253-268 (2015).

4. Ohinata, Y. et al. A signaling principle for the specification of the germ cell lineage in mice. Cell 137, 571-584 (2009).

5. Kobayashi, T. et al. Principles of early human development and germ cell program from conserved model systems. Nature 546, 416-420 (2017).

6. Irie, N., Sybirna, A. \& Surani, M. A. What can stem cell models tell us about human germ cell biology? Curr. Top. Dev. Biol. 129, 25-65 (2018).

7. Magnusdottir, E. et al. A tripartite transcription factor network regulates primordial germ cell specification in mice. Nat. Cell Biol. 15, 905-915 (2013).

8. Nakaki, F. et al. Induction of mouse germ-cell fate by transcription factors in vitro. Nature 501, 222-226 (2013).

9. Yamaji, M. et al. Critical function of Prdm14 for the establishment of the germ cell lineage in mice. Nat. Genet. 40, 1016-1022 (2008).

10. Okashita, N. et al. PRDM14 promotes active DNA demethylation through the ten-eleven translocation (TET)-mediated base excision repair pathway in embryonic stem cells. Development 141, 269-280 (2014).

11. Shirane, K. et al. Global landscape and regulatory principles of DNA methylation reprogramming for germ cell specification by mouse pluripotent stem cells. Dev. Cell 39, 87-103 (2016).

12. Burton, A. et al. Single-cell profiling of epigenetic modifiers identifies PRDM14 as an inducer of cell fate in the mammalian embryo. Cell Rep. 5, 687-701 (2013).

13. Chia, N. Y. et al. A genome-wide RNAi screen reveals determinants of human embryonic stem cell identity. Nature 468, 316-320 (2010).

14. Nakaki, F. \& Saitou, M. PRDM14: a unique regulator for pluripotency and epigenetic reprogramming. Trends Biochem. Sci. 39, 289-298 (2014).

15. Nady, N. et al. ETO family protein Mtgr1 mediates Prdm14 functions in stem cell maintenance and primordial germ cell formation. eLife 4, e10150 (2015).

16. Ma, Z., Swigut, T., Valouev, A., Rada-Iglesias, A. \& Wysocka, J. Sequencespecific regulator Prdm14 safeguards mouse ESCs from entering extraembryonic endoderm fates. Nat. Struct. Mol. Biol. 18, 120-127 (2011).

17. Chan, Y. S. et al. A PRC2-dependent repressive role of PRDM14 in human embryonic stem cells and induced pluripotent stem cell reprogramming. Stem Cells 31, 682-692 (2013).

18. Tsuneyoshi, N. et al. PRDM14 suppresses expression of differentiation marker genes in human embryonic stem cells. Biochem. Biophys. Res. Commun. 367, 899-905 (2008)

19. Sugawa, F. et al. Human primordial germ cell commitment in vitro associates with a unique PRDM14 expression profile. EMBO J. 34, 1009-1024 (2015).

20. Kojima, Y. et al. Evolutionarily distinctive transcriptional and signaling programs drive human germ cell lineage specification from pluripotent stem cells. Cell Stem Cell 21, 517-532.e5 (2017)

21. Hara, K. et al. Evidence for crucial role of hindgut expansion in directing proper migration of primordial germ cells in mouse early embryogenesis. Dev. Biol. 330, 427-439 (2009).

22. Kanai-Azuma, M. et al. Depletion of definitive gut endoderm in Sox17-null mutant mice. Development 129, 2367-2379 (2002)

23. Nishimura, K., Fukagawa, T., Takisawa, H., Kakimoto, T. \& Kanemaki, M. An auxin-based degron system for the rapid depletion of proteins in nonplant cells. Nat. Methods 6, 917-922 (2009).

24. Brosh, R. et al. A dual molecular analogue tuner for dissecting protein function in mammalian cells. Nat. Commun. 7, 11742 (2016).

25. Cong, L. et al. Multiplex genome engineering using CRISPR/Cas systems. Science 339, 819-823 (2013)

26. Tang, W. W. et al. A unique gene regulatory network resets the human germline epigenome for development. Cell 161, 1453-1467 (2015).

27. Kurimoto, K. et al. Complex genome-wide transcription dynamics orchestrated by Blimp1 for the specification of the germ cell lineage in mice. Genes Dev. 22, 1617-1635 (2008).

28. Capecchi, M. R. Gene targeting in mice: functional analysis of the mammalian genome for the twenty-first century. Nat. Rev. Genet. 6, 507-512 (2005).

29. Chen, Y. et al. Engineering human stem cell lines with inducible gene knockout using CRISPR/Cas9. Cell Stem Cell 17, 233-244 (2015).

30. Sacher, R., Stergiou, L. \& Pelkmans, L. Lessons from genetics: interpreting complex phenotypes in RNAi screens. Curr. Opin. Cell Biol. 20, 483-489 (2008).

31. Turlo, K. A., Gallaher, S. D., Vora, R., Laski, F. A. \& Iruela-Arispe, M. L. When Cre-mediated recombination in mice does not result in protein loss. Genetics 186, 959-967 (2010).

32. Natsume, T. \& Kanemaki, M. T. Conditional degrons for controlling protein expression at the protein level. Annu. Rev. Genet. 51, 83-102 (2017).

33. Wang, W. et al. Chromosomal transposition of PiggyBac in mouse embryonic stem cells. Proc. Natl Acad. Sci. USA 105, 9290-9295 (2008). 
34. Nora, E. P. et al. Targeted degradation of CTCF decouples local insulation of chromosome domains from genomic compartmentalization. Cell 169, 930-944.e22 (2017).

35. Morawska, M. \& Ulrich, H. D. An expanded tool kit for the auxin-inducible degron system in budding yeast. Yeast 30, 341-351 (2013).

36. Seguin, C. A., Draper, J. S., Nagy, A. \& Rossant, J. Establishment of endoderm progenitors by SOX transcription factor expression in human embryonic stem cells. Cell Stem Cell 3, 182-195 (2008).

37. Hayashi, K., Ohta, H., Kurimoto, K., Aramaki, S. \& Saitou, M. Reconstitution of the mouse germ cell specification pathway in culture by pluripotent stem cells. Cell 146, 519-532 (2011).

38. Haugwitz, M., Nourzaie, O., Gandlur, S. \& Sagawa, H. ProteoTuner: a novel system with rapid kinetics enables reversible control of protein levels in cells and organisms. BioTechniques 44, 432-433 (2008).

39. Banaszynski, L. A., Chen, L. C., Maynard-Smith, L. A., Ooi, A. G. \& Wandless, T. J. A rapid, reversible, and tunable method to regulate protein function in living cells using synthetic small molecules. Cell 126, 995-1004 (2006).

40. Yamaji, M. et al. PRDM14 ensures naive pluripotency through dual regulation of signaling and epigenetic pathways in mouse embryonic stem cells. Cell Stem Cell 12, 368-382 (2013).

41. Heinz, S. et al. Simple combinations of lineage-determining transcription factors prime cis-regulatory elements required for macrophage and B cell identities. Mol. Cell 38, 576-589 (2010).

42. Nichols, J. \& Smith, A. Naive and primed pluripotent states. Cell Stem Cell 4, 487-492 (2009).

43. Tauber, M. \& Fischle, W. Conserved linker regions and their regulation determine multiple chromatin-binding modes of UHRF1. Nucleus 6, 123-132 (2015).

44. Milagre, I. et al. Gender differences in global but not targeted demethylation in iPSC reprogramming. Cell Rep. 18, 1079-1089 (2017).

45. Tachibana, M. et al. Histone methyltransferases G9a and GLP form heteromeric complexes and are both crucial for methylation of euchromatin at H3-K9. Genes Dev. 19, 815-826 (2005).

46. Grabole, N. et al. Prdm14 promotes germline fate and naive pluripotency by repressing FGF signalling and DNA methylation. EMBO Rep. 14, 629-637 (2013).

47. Irie, N., Tang, W. W. \& Azim Surani, M. Germ cell specification and pluripotency in mammals: a perspective from early embryogenesis. Reprod. Med. Biol. 13, 203-215 (2014).

48. Nakamura, T. et al. A developmental coordinate of pluripotency among mice, monkeys and humans. Nature 537, 57-62 (2016).

49. Campolo, F. et al. Essential role of Sox 2 for the establishment and maintenance of the germ cell line. Stem Cells 31, 1408-1421 (2013).

50. Lin, I. Y. et al. Suppression of the SOX2 neural effector gene by PRDM1 promotes human germ cell fate in embryonic stem cells. Stem Cell Rep. 2, 189-204 (2014).

51. Boyer, L. A. et al. Core transcriptional regulatory circuitry in human embryonic stem cells. Cell 122, 947-956 (2005).

52. Wang, Z., Oron, E., Nelson, B., Razis, S. \& Ivanova, N. Distinct lineage specification roles for NANOG, OCT4, and SOX2 in human embryonic stem cells. Cell Stem Cell 10, 440-454 (2012).

53. Zhang, L., Ward, J. D., Cheng, Z. \& Dernburg, A. F. The auxin-inducible degradation (AID) system enables versatile conditional protein depletion in C. elegans. Development 142, 4374-4384 (2015).

54. Trost, M., Blattner, A. C. \& Lehner, C. F. Regulated protein depletion by the auxin-inducible degradation system in Drosophila melanogaster. Fly 10, 35-46 (2016).

55. Gell, J. J., Zhao, J., Chen, D., Hunt, T. J. \& Clark, A. T. PRDM14 is expressed in germ cell tumors with constitutive overexpression altering human germline differentiation and proliferation. Stem Cell Res. 27, 46-56 (2018).

56. Thomson, J. A. et al. Embryonic stem cell lines derived from human blastocysts. Science 282, 1145-1147 (1998).

57. Chen, G. et al. Chemically defined conditions for human iPSC derivation and culture. Nat. Methods 8, 424-429 (2011).

58. Schindelin, J. et al. Fiji: an open-source platform for biological-image analysis. Nat. Methods 9, 676-682 (2012).

59. Anastassiadis, K. et al. Dre recombinase, like Cre, is a highly efficient sitespecific recombinase in E-coli, mammalian cells and mice. Dis. Model Mech. 2, 508-515 (2009).

60. Andrews, S. FastQC: a quality control tool for high throughput sequence data. Babraham Bioinformatics, http://www.bioinformatics.babraham.ac.uk/ projects/fastqc (2010).

61. Krueger, F. Trim Galore!: A wrapper tool around Cutadapt and FastQC to consistently apply quality and adapter trimming to FastQ files. Babraham Bioinformatics, https://www.bioinformatics.babraham.ac.uk/projects/ trim_galore/ (2012).

62. Dobin, A. et al. STAR: ultrafast universal RNA-seq aligner. Bioinformatics 29, 15-21 (2013).
63. Jin, Y., Tam, O. H., Paniagua, E. \& Hammell, M. TEtranscripts: a package for including transposable elements in differential expression analysis of RNA-seq datasets. Bioinformatics 31, 3593-3599 (2015).

64. Love, M. I., Huber, W. \& Anders, S. Moderated estimation of fold change and dispersion for RNA-seq data with DESeq2. Genome Biol. 15, 550 (2014).

65. Subramanian, A. et al. Gene set enrichment analysis: a knowledge-based approach for interpreting genome-wide expression profiles. Proc. Natl Acad. Sci. USA 102, 15545-15550 (2005).

66. Huang da, W., Sherman, B. T. \& Lempicki, R. A. Systematic and integrative analysis of large gene lists using DAVID bioinformatics resources. Nat. Protoc. 4, 44-57 (2009).

67. Lin, G. et al. VennPainter: a tool for the comparison and identification of candidate genes based on venn diagrams. PLOS ONE 11, e0154315 (2016).

68. Polanski, K. et al. Wigwams: identifying gene modules co-regulated across multiple biological conditions. Bioinformatics 30, 962-970 (2014).

69. Csardi, G. \& Nepusz, T. The igraph software package for complex network research. InterJournal 1695, CX.18 (2006).

70. Ritchie, M. E. et al. limma powers differential expression analyses for RNAsequencing and microarray studies. Nucleic Acids Res. 43, e47, https://doi.org/ 10.1093/nar/gkv007 (2015).

71. Li, H. \& Durbin, R. Fast and accurate short read alignment with Burrows-Wheeler transform. Bioinformatics 25, 1754-1760 (2009)

72. Li, H. et al. The sequence alignment/map format and SAMtools. Bioinformatics 25, 2078-2079 (2009).

73. Zhang, Y. et al. Model-based analysis of ChIP-Seq (MACS). Genome Biol. 9 R137 (2008)

74. Liao, Y., Smyth, G. K. \& Shi, W. featureCounts: an efficient general purpose program for assigning sequence reads to genomic features. Bioinformatics $\mathbf{3 0}$, 923-930 (2014)

75. Ewels, P., Magnusson, M., Lundin, S. \& Kaller, M. MultiQC: summarize analysis results for multiple tools and samples in a single report. Bioinformatics 32, 3047-3048 (2016).

76. Quinlan, A. R. \& Hall, I. M. BEDTools: a flexible suite of utilities for comparing genomic features. Bioinformatics 26, 841-842 (2010).

77. Ramirez, F. et al. deepTools2: a next generation web server for deepsequencing data analysis. Nucleic Acids Res. 44, W160-W165 (2016)

78. Robinson, J. T. et al. Integrative genomics viewer. Nat. Biotechnol. 29, 24-26 (2011).

79. Yu, G., Wang, L. G. \& He, Q. Y. ChIPseeker: an R/Bioconductor package for ChIP peak annotation, comparison and visualization. Bioinformatics 31, 2382-2383 (2015)

80. Perez, A. C. \& Goossens, A. Jasmonate signalling: a copycat of auxin signalling? Plant Cell Environ. 36, 2071-2084 (2013).

\section{Acknowledgements}

We thank Dr Elphège Nora for kindly providing reagents (plasmids containing AID degron and TIR1 sequences) and guidance for the establishment of the auxininducible degron. We are grateful to Dr Richard Butler for writing the Fiji plugin for fluorescence intensity quantification. We gratefully acknowledge Dr Toshihiro Kobayashi, Dr Naoko Irie and Dr Ufuk Günesdogan for their useful advice throughou the project. A.S. was funded by the 4 -year Wellcome Trust PhD Scholarship; M.P.S. was funded by a Churchill scholarship; MAS holds a Wellcome Senior Investigator Award, and grant from the MRC. The Gurdon Institute is supported through core funding from the Wellcome Trust and Cancer Research UK. W.H.G. was supported by an EMBO long term fellowship (ALTF 263-2014). W.W.C.T. holds a Croucher Fellowship for Postdoctoral Research.

\section{Author contributions}

The study was conceived and designed by A.S. and M.A.S. A.S. performed most experiments; W.W.C.T. and S.D. performed most bioinformatics analyses; W.W.C.T. prepared human gonadal sections and conducted some IF stainings of human gonadal sections. M.P.S helped with revision experiments, including time-course hPGCLC induction for some IF experiments and time-course of TIR1-JAZ rescue. W.H.G. helped with ChIP experiments and data analysis. R.B. provided reagents for the JAZ degron and contributed JAZ degron sequence characterisation in HEK-293T cells. The study was supervised by M.A.S. The paper was written by A.S. and M.A.S. with contributions from most authors.

\section{Competing interests}

The authors declare no competing interests. 


\section{Additional information}

Supplementary information is available for this paper at https://doi.org/10.1038/s41467020-15042-0

Correspondence and requests for materials should be addressed to M.A.S.

Peer review information Nature Communications thanks Masato Kanemaki and the other, anonymous, reviewer(s) for their contribution to the peer review of this work. Peer reviewer reports are available.

Reprints and permission information is available at http://www.nature.com/reprints

Publisher's note Springer Nature remains neutral with regard to jurisdictional claims in published maps and institutional affiliations. (c) (i) Open Access This article is licensed under a Creative Commons Attribution 4.0 International License, which permits use, sharing, adaptation, distribution and reproduction in any medium or format, as long as you give appropriate credit to the original author(s) and the source, provide a link to the Creative Commons license, and indicate if changes were made. The images or other third party material in this article are included in the article's Creative Commons license, unless indicated otherwise in a credit line to the material. If material is not included in the article's Creative Commons license and your intended use is not permitted by statutory regulation or exceeds the permitted use, you will need to obtain permission directly from the copyright holder. To view a copy of this license, visit http://creativecommons.org/ licenses/by/4.0/.

(C) The Author(s) 2020 